TRANSACTIONS OF THE

AMERICAN MATHEMATICAL SOCIETY

Volume 357, Number 4, Pages 1417-1444

S 0002-9947(04)03521-4

Article electronically published on July 22, 2004

\title{
AN ITERATIVE CONSTRUCTION OF GORENSTEIN IDEALS
}

\author{
C. BOCCI, G. DALZOTTO, R. NOTARI, AND M. L. SPREAFICO
}

\begin{abstract}
In this paper, we present a method to inductively construct Gorenstein ideals of any codimension $c$. We start from a Gorenstein ideal $I$ of codimension $c$ contained in a complete intersection ideal $J$ of the same codimension, and we prove that under suitable hypotheses there exists a new Gorenstein ideal contained in the residual ideal $I: J$. We compare some numerical data of the starting and the resulting Gorenstein ideals of the construction. We compare also the Buchsbaum-Eisenbud matrices of the two ideals, in the codimension three case. Furthermore, we show that this construction is independent from the other known geometrical constructions of Gorenstein ideals, providing examples.
\end{abstract}

\section{INTRODUCTION}

A closed scheme $X \subset \mathbb{P}^{n}$ defined by the saturated homogeneous ideal $I_{X} \subset R:=$ $K\left[x_{0}, \ldots, x_{n}\right]$ is said to be arithmetically Gorenstein if its coordinate ring $R / I_{X}$ is a Gorenstein ring. Arithmetically Gorenstein schemes are a generalization of complete intersection rings, and indeed the two notions coincide in codimension 2.

The codimension 3 Gorenstein rings are completely described from an algebraic point of view by the structure theorem of Buchsbaum and Eisenbud in [3], while in higher codimension no structure theorem is known. Nevertheless, a geometric description of arithmetically Gorenstein schemes of any codimension is not understood. For this reason, many authors give geometric constructions of some particular families of arithmetically Gorenstein schemes (cf. [19, [12, 15]). These constructions are successfully applied in the context of Gorenstein liaison to produce Gorenstein links of given schemes and to study the Gorenstein liaison classes of some particular schemes (see, for example, [12], 11], [5], 6], 77, [17, 13]).

A rather natural but naive question is if it is possible to use complete intersection schemes to produce arithmetically Gorenstein schemes. In the present paper, we show that if an arithmetically Gorenstein scheme $V$ contains a complete intersection subscheme $W$ of the same codimension, then, under suitable hypotheses, it is possible to replace $W$ with a complete intersection scheme $W^{\prime}$ in such a way that $(V \backslash W) \cup W^{\prime}$ is still arithmetically Gorenstein (see Theorem 3.2, below). Then, our construction starts with an arithmetically Gorenstein scheme and produces a new arithmetically Gorenstein scheme of the same codimension, but with different minimal free resolution (in general, the two ideals have a different number of generators). For this reason, we consider this construction as iterative. Furthermore,

Received by the editors February 24, 2003 and, in revised form, September 26, 2003.

2000 Mathematics Subject Classification. Primary 14M05, 13H10; Secondary 14M06, 13D02, $18 \mathrm{G} 10$. 
we work on ideals more than on schemes, and so our construction can be used in the Artinian case, too. Of course, the starting ideal can be a complete intersection ideal, as well. Then, our construction shows that it is possible to obtain arithmetically Gorenstein schemes starting from complete intersection ones. Our hope is that such a construction will be successfully applied to study Gorenstein liaison and to give new insight to obtain a classification result for arithmetically Gorenstein rings of codimension $\geq 4$.

The plan of the paper is the following. In Section 2, we describe the residual ideal of a complete intersection ideal with respect to a Gorenstein ideal. In fact, $V \backslash W$ is the residual scheme to $W$ with respect to $V$. Furthermore, in the same section, we investigate in some detail the case where $V \backslash W$ is a complete intersection scheme.

In Section 3, we prove the main result of the paper, Theorem 3.2 where we prove that $(V \backslash W) \cup W^{\prime}$ is arithmetically Gorenstein . The idea of the proof is very simple and consists in producing a free resolution of the ideal we are interested in. This result is achieved by using the resolutions of the various ideals involved, and by carefully analyzing the final resolution. The main tool is homological algebra. In this section, we also prove what are the relations among the Hilbert functions of the ideals involved in the construction. At the end, as an application of Theorem 3.2, we show that every reduced arithmetically Gorenstein zero-dimensional closed scheme $X \subset \mathbb{P}^{n}$ of degree $\operatorname{deg} X=n+2$ can be obtained from the described iterative construction.

Section 4, which was the first case we investigated, is devoted to the codimension 3 case. There, we show the relation between the Buchsbaum-Eisenbud matrices of the input and output Gorenstein ideals of the construction. The main result of the section is a characterization of the Gorenstein ideals which are output of the iterative construction, via their Buchsbaum-Eisenbud matrix. In Section 5, we compare various geometrical constructions of Gorenstein ideals. Using the characterization obtained in Section 4, we exhibit examples of codimension 3 schemes arising from some of the known constructions but not from ours, and conversely.

\section{The Residual IDEAL}

In this section we recall some basic definitions used in the paper, and we study the residual ideal to a complete intersection ideal $J$ with respect to a Gorenstein ideal $I$. In particular, we compute the generators of the residual ideal, obtaining very precise results in the case where $I$ is a complete intersection.

All the ideals we consider in this paper are homogeneous ideals in the graded polynomial ring $R=K\left[x_{0}, \ldots, x_{n}\right]$, where $K$ is an algebraically closed field, and all the $x_{i}{ }^{\prime}$ s have degree 1 .

To start with, we introduce the Cohen-Macaulay ideals.

Definition 2.1. Let $I \subset R$ be a homogeneous ideal. $I$ is a Cohen-Macaulay ideal if $\operatorname{dim}(R / I)=\operatorname{depth}(R / I)$. The codimension of $I$ is $c=\operatorname{dim}(R)-\operatorname{dim}(R / I)$. Equivalently, we say that $R / I$ is a Cohen-Macaulay ring, and the subscheme of the projective space that it defines is an arithmetically Cohen-Macaulay (aCM, for short) subscheme.

Remark 2.2. If $I$ is a Cohen-Macaulay ideal of codimension $c$, the minimal free resolution of $R / I$ has length $c$, i.e., the minimal free resolution of $R / I$ has the form

$$
0 \rightarrow F_{c} \longrightarrow F_{c-1} \longrightarrow \ldots \longrightarrow F_{1} \longrightarrow R \longrightarrow R / I \rightarrow 0
$$

where $F_{i}$ is a free $R$-module, for every $i$. 
Now, we define a particular class of Cohen-Macaulay ideals, the Gorenstein ideals, which are what we are interested in.

Definition 2.3. Let $I \subset R$ be a homogeneous ideal. $I$ is a Gorenstein ideal of codimension $c$ if $I$ is a Cohen-Macaulay ideal of codimension $c$ and $F_{c} \cong R\left(-t_{I}\right)$ for some integer $t_{I}$, that is to say, $F_{c}$ has rank 1 . Equivalently, we say that the quotient ring $R / I$ is Gorenstein, and the subscheme of the projective space that it defines is an arithmetically Gorenstein (aG, for short) subscheme.

Now, we stress some well known properties of Gorenstein ideals.

Proposition 2.4 ([14, Proposition 4.1.1). Let $I \subset R$ be a homogeneous ideal of codimension $c$. Then, the following are equivalent:

(1) I is Gorenstein.

(2) The minimal free resolution (1) of $R / I$ is self-dual up to twisting by $n+1$.

(3) $R / I \cong \operatorname{Ext}_{\mathrm{R}}^{c}(R / I, R)(l-n-1)$ for some $l \in \mathbb{Z}$.

At last, we define the complete intersection ideals, which are particular Gorenstein ideals.

Definition 2.5. Let $J \subset R$ be a homogeneous ideal minimally generated by the forms $g_{1}, \ldots, g_{c} . J$ is a complete intersection ideal of codimension $c$ if

$$
\left(g_{1}, \ldots, g_{i-1}\right): g_{i} R=\left(g_{1}, \ldots, g_{i-1}\right) \text { for } i=2, \ldots, c .
$$

It is possible to characterize the complete intersection ideals via their minimal free resolutions.

Proposition 2.6 (14, Example 1.5.1). Let $J \subset R$ be a homogeneous ideal minimally generated by $g_{1}, \ldots, g_{c} . J$ is a complete intersection ideal of codimension $c$ if, and only if, a minimal free resolution of $R / J$ is

$$
0 \rightarrow \wedge^{c} G \longrightarrow \wedge^{c-1} G \longrightarrow \ldots \longrightarrow \wedge^{2} G \longrightarrow G \longrightarrow R \longrightarrow R / J \rightarrow 0,
$$

where $G=\bigoplus_{i=1}^{c} R\left(-\operatorname{deg} g_{i}\right)$.

The free $R$-module $G$ has rank $c$, and so $\wedge^{c} G$ is a rank 1 free $R$-module isomorphic to $R\left(-\sum_{i=1}^{c} \operatorname{deg} g_{i}\right)$. Then we have

Corollary 2.7. A complete intersection ideal $J \subset R$ is Gorenstein, and $t_{J}=$ $\sum_{i=1}^{c} \operatorname{deg} g_{i}$.

Now, we recall the definition of linked ideals from liaison theory.

Definition 2.8 ([14], Definition 5.1.2). Let $I, J_{1}, J_{2} \subset R$ be homogeneous, unmixed ideals of the same codimension $c$. We say that $J_{1}, J_{2}$ are (directly) CI-linked (resp. $G$-linked) by the complete intersection (resp. Gorenstein) ideal $I$ if $I \subset J_{1} \cap J_{2}$ and

$$
I: J_{1}=J_{2} \quad \text { and } \quad I: J_{2}=J_{1} .
$$

Moreover, we say that $J_{2}$ is the residual ideal to $J_{1}$ with respect to the linking ideal $I$.

We want to study in detail the residual ideal to a complete intersection ideal $J$ with respect to a Gorenstein ideal $I$. With this aim, let $I, J \subset R$ be a Gorenstein 
ideal and a complete intersection ideal, respectively, and let $I \subset J$. Hence, we have a map of complexes, induced by the inclusion

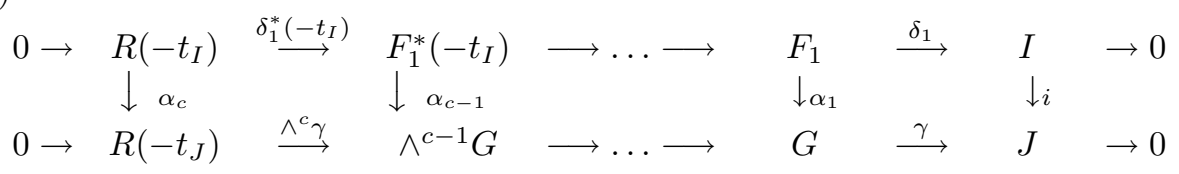

where $F_{1}^{*}=\operatorname{Hom}\left(F_{1}, R\right)$, and $\alpha^{*}: Q^{*} \rightarrow P^{*}$ is the natural dual map, defined as $\alpha^{*}(t)=\alpha \circ t$. Observe that in Proposition 2.4, by the dual of a $R$-module $F$ we mean $\operatorname{Hom}(F, R(-n-1))$.

For completeness, we prove some properties of the ideal $I_{\text {res }}=I: J$, by specializing to our case some well known results.

Lemma 2.9. The ideal $I_{\mathrm{res}}=I: J$ is unmixed of codimension $c$, and $I_{\mathrm{res}}=I+f R$ where $f$ is a form of degree $t_{I}-t_{J}$ which represents the map $\alpha_{c}$ of diagram (3).

Proof. By [14, Proposition 5.2.3, the residual ideal $I_{\text {res }}=I: J$ is unmixed of codimension $c$, and the ideals $J$ and $I_{\text {res }}$ are linked by the Gorenstein ideal $I$.

To show that $I_{\text {res }}=I+f R$ we follow the proof of a result by C. Peskine and L. Szpiro (14], Proposition 5.2.10). The resolution of $\operatorname{Ext}_{R}^{c}\left(R / I_{\mathrm{res}}, R\right)\left(-t_{I}\right)$ is obtained by a mapping cone from the complex (3), and the last map of this resolution is then

$$
\left(\begin{array}{c}
(-1)^{c+1} \alpha_{c} \\
\delta_{1}^{*}\left(-t_{I}\right)
\end{array}\right): R\left(-t_{I}\right) \rightarrow \begin{gathered}
R\left(-t_{J}\right) \\
F_{1}^{*}\left(-t_{I}\right)
\end{gathered} .
$$

As explained in the proof of [14, Proposition 5.2.10, a free resolution of $I_{\text {res }}$ can be produced by applying $\operatorname{Hom}_{R}(-, R)$ to the resolution of $\operatorname{Ext}_{R}^{c}\left(R / I_{\text {res }}, R\right)\left(-t_{I}\right)$, and so we get

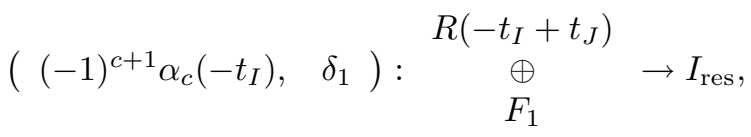

which proves the claim.

Remark 2.10. Of course, the map $\alpha_{c}$ is determined up to homotopy. In our situation, this means that $f \bmod I$ is unique up to invertible elements.

Furthermore, a free resolution (not necessarily minimal) of $I_{\text {res }}$ can be obtained by a mapping cone from the free resolutions of $I$ and $J$ via the short exact sequence

$$
0 \rightarrow J\left(t_{J}-t_{I}\right) \longrightarrow I \oplus f R \longrightarrow I_{\mathrm{res}} \rightarrow 0 .
$$

The exactness of this sequence stems from the following lemma.

Lemma 2.11. $I \cap f R=J \cdot f R$.

Proof. It is obvious that $J \cdot f R \subseteq I \cap f R$. To prove the inverse inclusion, we consider a homogeneous form $h$ such that $h f \in I$. Of course, $h g \in I$ for every $g \in I$. Then, $h \in I: I_{\mathrm{res}}=J$, and so $h f \in J \cdot f R$.

From the map of complexes (3), we deduce a relation among the various maps.

Proposition 2.12. The map $\alpha_{c}$ satisfies the relation

$$
\wedge^{c} \gamma \circ \alpha_{c}=\alpha_{c-1} \circ \alpha_{1}^{*}\left(-t_{I}\right) \circ \gamma^{*}\left(-t_{I}\right),
$$

where $\alpha_{1}^{*}: G^{*} \rightarrow F_{1}^{*}$ is the map dual to $\alpha_{1}$. 
Proof. From the map of complexes (3), we get the equalities

$$
\wedge^{c} \gamma \circ \alpha_{c}=\alpha_{c-1} \circ \delta_{1}^{*}\left(-t_{I}\right) \text { and } \delta_{1}=\gamma \circ \alpha_{1} .
$$

If we compute the dual maps to the maps of the second equality and twist them for suitable integers, we get

$$
\delta_{1}^{*}\left(-t_{I}\right)=\alpha_{1}^{*}\left(-t_{I}\right) \circ \gamma^{*}\left(-t_{I}\right) .
$$

Hence, the claim follows by substitution in the first of the two equalities.

Corollary 2.13. Let $\overline{\alpha_{c}}: \wedge^{c-1} G\left(t_{J}-t_{I}\right) \rightarrow \wedge^{c-1} G$ be the map which acts as $\alpha_{c}$ on each free addendum of $\wedge^{c-1} G$. Then,

$$
\overline{\alpha_{c}} \circ \wedge^{c} \gamma\left(t_{J}-t_{I}\right)=\alpha_{c-1} \circ \alpha_{1}^{*}\left(-t_{I}\right) \circ \gamma^{*}\left(-t_{I}\right) .
$$

Proof. By its definition, it is evident that

$$
\overline{\alpha_{c}} \circ \wedge^{c} \gamma\left(t_{J}-t_{I}\right)=\wedge^{c} \gamma \circ \alpha_{c},
$$

and so we have the claim.

Now, we consider the special case when $I$ is a complete intersection.

Proposition 2.14. If I is a complete intersection ideal, the residual ideal $I_{\mathrm{res}}$ is equal to $I+(\operatorname{det} A) R$, where $A$ is a square matrix which represents the map $\alpha_{1}$.

Proof. Since $I$ is a complete intersection ideal, its minimal free resolution is Koszul, and so $F_{j}=\wedge^{j} F_{1}$ for $j=1, \ldots, c$. Moreover, for the map of complexes, we have $\alpha_{j}=\wedge^{j} \alpha_{1}$, and so $\alpha_{c}=\wedge^{c} \alpha_{1}$. Hence, the map $\alpha_{c}$ is represented by $\operatorname{det} A$ for whatever matrix $A$ represents the map $\alpha_{1}$.

In this last case, it is possible that the residual ideal $I_{\text {res }}$ is a complete intersection, too. In fact, we have

Proposition 2.15. Let $J$ be generated by $g_{1}, \ldots, g_{c}$. Then, $I_{\text {res }}$ is a complete intersection if, and only if, $I$ is generated by $g_{1}, \ldots, g_{c-1}, g_{c} \operatorname{det} A$, up to reordering the generators of $J$.

Proof. Let $I, J$ be generated by $f_{1}, \ldots, f_{c}$ and $g_{1}, \ldots, g_{c}$, respectively. Let $A$ be a matrix which represents the map $\alpha_{1}$ in such a way that $\left(f_{1}, \ldots, f_{c}\right)=\left(g_{1}, \ldots, g_{c}\right) A$. By Proposition 2.14 $I_{\text {res }}$ is generated by $f_{1}, \ldots, f_{c}$, $\operatorname{det} A$.

The residual ideal $I_{\text {res }}$ is a complete intersection if, and only if, one of the generators is in the ideal generated by the remaining ones.

If $\operatorname{det} A \in I$, then $I: J=I$, but this is not possible by comparing the degrees of the involved ideals. Hence, after suitably choosing and reordering the generators of $I$, we can suppose that $f_{c}=\operatorname{det} A f_{c}^{\prime}$.

Moreover, we have the following commutative diagram from the sequence (4):

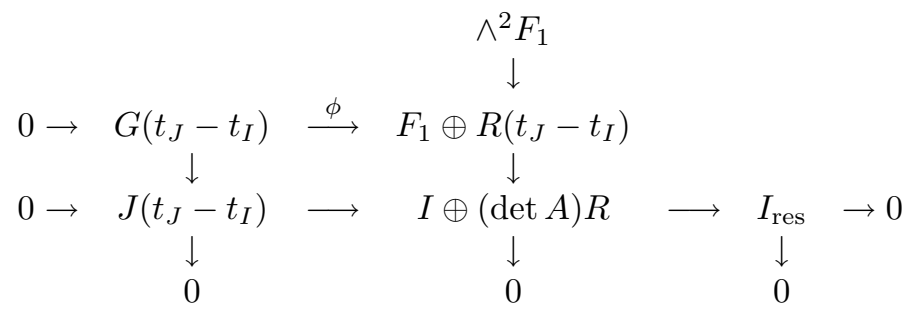


where $\phi=\left(\alpha_{c-1}^{*}\left(-t_{I}\right), \gamma\left(t_{J}-t_{I}\right)\right)$. But $\alpha_{c-1}=\wedge^{c-1} \alpha_{1}$, and so $\alpha_{c-1}^{*}$ is represented by the adjoint matrix $\operatorname{adj}(A)$ of $A$.

The resolution we get by the mapping cone procedure is not minimal. Hence, an entry of $\operatorname{adj}(A)$ is a non-zero constant, and so there exists a $(c-1) \times(c-1)$ submatrix of $A$ whose determinant is in $K$ and is non-zero. Then, up to suitable choice and re-ordering of the generators of $I$ and $J$, we can say that $I$ is generated by $g_{1}, \ldots, g_{c-1}, g_{c} \operatorname{det} A$.

\section{The iterative construction of Gorenstein ideals}

In this section, given a Gorenstein ideal $I$ contained in a complete intersection ideal $J$ of the same codimension $c$, we construct a new Gorenstein ideal of codimension $c$ with a minimal set of generators of cardinality different from that of $I$. The geometric idea of the construction was explained in the Introduction.

We use the same notation as in Section 2.

Let $J \subset R$ be a complete intersection ideal of codimension $c$ minimally generated by $g_{1}, g_{2}, \ldots, g_{c}$, and let $I \subset J$ be a homogeneous Gorenstein ideal of the same codimension, minimally generated by $g_{1}, f_{1}, \ldots, f_{k}$.

Remark 3.1. Up to a suitable choice of the generators of $I$, we can assume that $f_{i} \in\left(g_{2}, \ldots, g_{c}\right)$ for each $i=1, \ldots, k$.

Theorem 3.2 (Construction of the Gorenstein ideal). Let $f$ be a form which represents the map $\alpha_{c}$ (cf. diagram (3) and Remark 2.10). We set

$$
\mathfrak{A}=\left(g_{1} g_{2}, \ldots, g_{1} g_{c}, f_{1}, \ldots, f_{k}, f+g_{1} g\right),
$$

where $g \in[R]_{t_{I}-t_{J}-\operatorname{deg}\left(g_{1}\right)}$ or $g=0$.

If $f+g_{1} g, g_{2}, \ldots, g_{c}$ is a regular sequence, then $\mathfrak{A}$ is a codimension c Gorenstein ideal.

To prove the result we shall construct a free resolution of the ideal $\mathfrak{A}$. For the convenience of the reader, we divide the proof into lemmas.

Notation. We set $\mathfrak{B}=\left(g_{1} g_{2}, \ldots, g_{1} g_{c}, f_{1}, \ldots, f_{k}\right)$, and $J^{\prime}=\left(g_{2}, \ldots, g_{c}\right)$.

Lemma 3.3. Under the hypotheses of Theorem 3.2,

$$
\mathfrak{B} \cap\left(f+g_{1} g\right) R=\left(f+g_{1} g\right) J^{\prime} .
$$

Proof. Let $F\left(f+g_{1} g\right) \in \mathfrak{B} \subset J^{\prime}$ (see Remark 3.1). Hence, $F \in J^{\prime}:\left(f+g_{1} g\right) R=J^{\prime}$; that is, $F\left(f+g_{1} g\right) \in\left(f+g_{1} g\right) J^{\prime}$.

Conversely, we have to show that $g_{i}\left(f+g_{1} g\right) \in \mathfrak{B}$, for $i=2, \ldots, c$, or equivalently, that $f g_{i} \in \mathfrak{B}$. From the map of complexes (3) we know that $\wedge^{c} \gamma \circ \alpha_{c}=\alpha_{c-1} \circ$ $\delta_{1}^{*}\left(-t_{I}\right)$, and so $f g_{i} \in I=\left(f_{1}, \ldots, f_{k}, g_{1}\right)$ for each $i=2, \ldots, c$. Then, we have $f g_{i}=h_{i 1} f_{1}+\cdots+h_{i k} f_{k}+h_{i} g_{1}$. By Remark 3.1, $f_{i} \in J^{\prime}$, and so $h_{i} g_{1} \in J^{\prime}$. The sequence $g_{1}, \ldots, g_{c}$ is regular, and so $h_{i} \in J^{\prime}$ for $i=2, \ldots, c$. Hence, $f g_{i} \in \mathfrak{B}$, and the claim follows.

As a consequence, we get

Corollary 3.4. The sequence

$$
0 \rightarrow J^{\prime}\left(-t_{I}+t_{J}\right) \stackrel{\psi}{\longrightarrow} \mathfrak{B} \oplus\left(f+g_{1} g\right) R \rightarrow \mathfrak{A} \rightarrow 0
$$

is exact, where $\psi(a)=\left(a\left(f+g_{1} g\right),-a\left(f+g_{1} g\right)\right)$. 
The sequence (6) allows us to produce a free resolution of $\mathfrak{A}$ via a mapping cone. At first, we compute a free resolution of $J^{\prime}$ and $\mathfrak{B}$.

Notation. The ideal $J^{\prime}$ is a complete intersection ideal, and its minimal free resolution is

$$
0 \rightarrow \wedge^{c-1} P \rightarrow \wedge^{c-2} P \rightarrow \cdots \rightarrow \wedge^{2} P \rightarrow P \stackrel{\Gamma}{\longrightarrow} J^{\prime} \rightarrow 0
$$

where $P=\bigoplus_{i=2}^{c} R\left(-\operatorname{deg} g_{i}\right)$, and the maps are $\wedge^{j} \Gamma$.

Remark 3.5. The minimal free resolution of the ideal $J=\left(g_{1}, g_{2}, \ldots, g_{c}\right)$ (see (2) ) is related to that of $J^{\prime}$ by the following:

$$
\wedge^{h} G \cong \wedge^{h} P \oplus \wedge^{h-1} P\left(-\operatorname{deg} g_{1}\right) .
$$

Let

$$
\pi_{h}: \wedge^{h} G \rightarrow \wedge^{h-1} P\left(-\operatorname{deg} g_{1}\right)
$$

be the projection, and let $\beta_{h}: F_{h} \rightarrow \wedge^{h-1} P\left(-\operatorname{deg} g_{1}\right)$ be equal to $\beta_{h}=\pi_{h} \circ \alpha_{h}$, $h=1, \ldots, c$. We can project the map of complexes (3), and so we have the following:

$$
\begin{array}{ccccccc}
0 \rightarrow & F_{c} & \stackrel{\delta_{c}}{\longrightarrow} & \ldots & F_{2} & \stackrel{\delta_{2}}{\longrightarrow} & F_{1} \\
& \downarrow_{\beta_{c}} & & & \downarrow_{\beta_{2}} & & \downarrow_{\beta_{1}} \\
0 \rightarrow & \wedge^{c-1} P\left(-\operatorname{deg} g_{1}\right) & \longrightarrow & \ldots & P\left(-\operatorname{deg} g_{1}\right) & \stackrel{\Gamma^{\prime}}{\longrightarrow} & R\left(-\operatorname{deg} g_{1}\right)
\end{array}
$$

where $\Gamma^{\prime}=\Gamma\left(-\operatorname{deg} g_{1}\right)$ and

$$
\beta_{j-1} \circ \delta_{j}=\wedge^{j-1} \Gamma^{\prime} \circ \beta_{j}
$$

By Remark 3.1 and from the choice of the generators of $I$, we have

$$
F_{1}=\bigoplus_{i=1}^{k} R\left(-\operatorname{deg} f_{i}\right) \oplus R\left(-\operatorname{deg} g_{1}\right)=\tilde{F}_{1} \oplus R\left(-\operatorname{deg} g_{1}\right)
$$

and furthermore, $\beta_{1}: \tilde{F}_{1} \oplus R\left(-\operatorname{deg} g_{1}\right) \rightarrow R\left(-\operatorname{deg} g_{1}\right)$ is $\left(0,1_{R\left(-\operatorname{deg} g_{1}\right)}\right)$.

Lemma 3.6. A free resolution of $\mathfrak{B}$ is

$$
0 \rightarrow H_{c} \stackrel{\varepsilon_{c}}{\longrightarrow} H_{c-1} \stackrel{\varepsilon_{c-1}}{\longrightarrow} \cdots \stackrel{\varepsilon_{2}}{\longrightarrow} H_{1} \stackrel{\varepsilon_{1}}{\longrightarrow} \mathfrak{B} \rightarrow 0,
$$

where

$$
H_{1}=P\left(-\operatorname{deg} g_{1}\right) \oplus \tilde{F}_{1},
$$

and

$$
H_{j}=\wedge^{j} P\left(-\operatorname{deg} g_{1}\right) \oplus F_{j}, \quad j=2, \ldots, c,
$$

while

$$
\varepsilon_{1}=\left(g_{1} \Gamma^{\prime}, \delta_{1 \mid \tilde{F}_{1}}\right), \quad \varepsilon_{2}=\left(\begin{array}{cc}
\wedge^{2} \Gamma^{\prime} & \beta_{2} \\
0 & \tilde{\delta}_{2}
\end{array}\right),
$$

where $\tilde{\delta_{2}}=\operatorname{proj}\left(F_{1} \rightarrow \tilde{F}_{1}\right) \circ \delta_{2}$ and

$$
\varepsilon_{j}=\left(\begin{array}{cc}
\wedge^{j} \Gamma^{\prime} & (-1)^{j} \beta_{j} \\
0 & \delta_{j}
\end{array}\right), \quad j=3, \ldots, c .
$$


Proof. $H_{1}$ and $\varepsilon_{1}$ are well understood, and easily computed. Now, we want to compute generators for the first syzygies of $\mathfrak{B}$.

Of course, the first syzygies of $J^{\prime}$ are syzygies of the first $c-1$ generators of $\mathfrak{B}$, up to twist by $-\operatorname{deg} g_{1}$, and so we have $\operatorname{rank}\left(\wedge^{2} P\right)$ first syzygies of $\mathfrak{B}$, and a map

$$
\wedge^{2} P\left(-\operatorname{deg} g_{1}\right) \stackrel{\left(\begin{array}{c}
\wedge^{2} \Gamma^{\prime} \\
0
\end{array}\right)}{\longrightarrow} P\left(-\operatorname{deg} g_{1}\right) \oplus \tilde{F}_{1} .
$$

Claim 1. The first syzygies of $I$ can be extended to syzygies of $\mathfrak{B}$.

If $\left(s_{1}, \ldots, s_{k}, s\right)^{t}$ is a syzygy of $\left(f_{1}, \ldots, f_{k}, g_{1}\right)$, then we have

$$
s_{1} f_{1}+\cdots+s_{k} f_{k}+s g_{1}=0 .
$$

By Remark [3.1, $f_{i} \in J^{\prime}$, and so $s g_{1} \in J^{\prime} . J^{\prime}$ is a complete intersection ideal, and so $s \in J^{\prime}$. If $x \in F_{2}$ is such that $\delta_{2}(x)=\left(s_{1}, \ldots, s_{k}, s\right)^{t}$, then $\beta_{1} \circ \delta_{2}(x)=s$. By (9), $\Gamma^{\prime} \circ \beta_{2}(x)=s$, and so $\beta_{2}(x)$ extends $\delta_{2}(x)$ to a syzygy of $\mathfrak{B}$. Then we can define the map

$$
F_{2} \stackrel{\left(\begin{array}{c}
\beta_{2} \\
\tilde{\delta_{2}}
\end{array}\right)}{\longrightarrow} P\left(-\operatorname{deg} g_{1}\right) \oplus \tilde{F}_{1} .
$$

Claim 2. The images of the two maps generate the first syzygy module of $\mathfrak{B}$.

If $\left(s_{2}^{\prime}, \ldots, s_{c}^{\prime}, s_{1}, \ldots, s_{k}\right)^{t}$ is a syzygy of $\mathfrak{B}$, then we have

$$
s_{2}^{\prime} g_{1} g_{2}+\cdots+s_{c}^{\prime} g_{1} g_{c}+s_{1} f_{1}+\cdots+s_{k} f_{k}=0,
$$

and so $\left(s_{1}, \ldots, s_{k}, s_{2}^{\prime} g_{2}+\cdots+s_{c}^{\prime} g_{c}\right)^{t}$ is a first syzygy of $I$. Hence, there exists $x \in F_{2}$ such that $\delta_{2}(x)=\left(s_{1}, \ldots, s_{k}, s_{2}^{\prime} g_{2}+\cdots+s_{c}^{\prime} g_{c}\right)^{t}$. Then,

$$
\left(s_{2}^{\prime}, \ldots, s_{c}^{\prime}, s_{1}, \ldots, s_{k}\right)^{t}-\left(\begin{array}{c}
\beta_{2} \\
\tilde{\delta}_{2}
\end{array}\right)(x)
$$

is a syzygy of the first $c-1$ generators of $\mathfrak{B}$, i.e., it is a syzygy of $J^{\prime}$. Hence, there exists $x^{\prime} \in \wedge^{2} P\left(-\operatorname{deg} g_{1}\right)$ such that

$$
\left(s_{2}^{\prime}, \ldots, s_{c}^{\prime}, s_{1}, \ldots, s_{k}\right)^{t}-\left(\begin{array}{c}
\beta_{2} \\
\tilde{\delta_{2}}
\end{array}\right)(x)=\left(\begin{array}{c}
\wedge^{2} \Gamma^{\prime} \\
0
\end{array}\right)\left(x^{\prime}\right),
$$

and so we have the claim.

Then

$$
H_{2}=\wedge^{2} P\left(-\operatorname{deg} g_{1}\right) \oplus F_{2}
$$

and

$$
\varepsilon_{2}=\left(\begin{array}{cc}
\wedge^{2} \Gamma^{\prime} & \beta_{2} \\
0 & \tilde{\delta_{2}}
\end{array}\right)
$$

Now, we compute the kernel of $\varepsilon_{2}$.

Of course, the second syzygies of $J^{\prime}$ are in the kernel of $\varepsilon_{2}$, and so we can define a map

$$
\wedge^{3} P\left(-\operatorname{deg} g_{1}\right) \stackrel{\left(\begin{array}{c}
\wedge^{3} \Gamma^{\prime} \\
0
\end{array}\right)}{\longrightarrow} \wedge^{2} P\left(-\operatorname{deg} g_{1}\right) \oplus F_{2} .
$$


Claim 3. The second syzygies of I can be lifted to second syzygies of $\mathfrak{B}$.

To prove this, let $x \in F_{3}$ be a second syzygy of $I$. Now, we prove that $\left(\left(-\beta_{3}\right)(x), \delta_{3}(x)\right)^{t} \in \operatorname{ker} \varepsilon_{2}$, i.e.,

$$
\left(\begin{array}{cc}
\wedge^{2} \Gamma^{\prime} & \beta_{2} \\
0 & \tilde{\delta}_{2}
\end{array}\right)\left(\begin{array}{c}
\left(-\beta_{3}\right)(x) \\
\delta_{3}(x)
\end{array}\right)=0
$$

By (9),$\left(\wedge^{2} \Gamma^{\prime} \circ\left(-\beta_{3}\right)+\beta_{2} \circ \delta_{3}\right)(x)=\left(-\beta_{2} \circ \delta_{3}+\beta_{2} \circ \delta_{3}\right)(x)=0$. Also, $\tilde{\delta_{2}} \circ \delta_{3}(x)=0$ because $\delta_{2} \circ \delta_{3}=0$ and by the definition of $\tilde{\delta_{2}}$.

Hence, we can define the map $\varepsilon_{3}: H_{3} \rightarrow H_{2}$ as

$$
\varepsilon_{3}=\left(\begin{array}{cc}
\wedge^{3} \Gamma^{\prime} & -\beta_{3} \\
0 & \delta_{3}
\end{array}\right)
$$

where $H_{3}=\wedge^{3} P\left(-\operatorname{deg} g_{1}\right) \oplus F_{3}$.

Claim 4. $\operatorname{Im} \varepsilon_{3}=\operatorname{ker} \varepsilon_{2}$.

Let $\left(y^{\prime}, y\right)^{t} \in \operatorname{ker} \varepsilon_{2}$. Then, we have the following relations:

$$
\tilde{\delta_{2}}(y)=0, \quad \wedge^{2} \Gamma^{\prime}\left(y^{\prime}\right)+\beta_{2}(y)=0 .
$$

First, we compute $\delta_{2}(y)$. To this aim, we recall that $\delta_{2}=\left(\begin{array}{c}\tilde{\delta_{2}} \\ \beta_{1} \circ \delta_{2}\end{array}\right)$. Of course, we have $\delta_{1} \circ \delta_{2}=0$, and so, explicitly,

$$
g_{1} \beta_{1} \circ \delta_{2}(y)+\delta_{1 \mid \tilde{F}_{1}} \circ \tilde{\delta}_{2}(y)=0 .
$$

By using the previous relations, we get $g_{1} \beta_{1} \circ \delta_{2}(y)=0$, and so $\beta_{1} \circ \delta_{2}(y)=0$. Therefore, $\delta_{2}(y)=0$. Hence there exists $x \in F_{3}$ such that $\delta_{3}(x)=y$. By construction, $\left(-\beta_{3}(x), \delta_{3}(x)\right)^{t} \in \operatorname{ker} \varepsilon_{2}$, and so $\left(y^{\prime}+\beta_{3}(x), 0\right)^{t} \in \operatorname{ker} \varepsilon_{2}$, i.e., $\wedge^{2} \Gamma^{\prime}\left(y^{\prime}+\beta_{3}(x)\right)=0$. Then, there exists $x^{\prime} \in \wedge^{3} P\left(-\operatorname{deg} g_{1}\right)$ such that $\wedge^{3} \Gamma^{\prime}\left(x^{\prime}\right)=y^{\prime}+\beta_{3}(x)$. Summarizing the results, we get that $\varepsilon_{3}\left(\left(x^{\prime}, x\right)^{t}\right)=$ $\left(y^{\prime}, y\right)^{t}$, and so we have computed the second syzygy module, too.

Now, we can go on by induction. We assume that $H_{j}=\wedge^{j} P\left(-\operatorname{deg} g_{1}\right) \oplus F_{j}$ and that $\varepsilon_{j}: H_{j} \rightarrow H_{j-1}$ is defined as

$$
\varepsilon_{j}=\left(\begin{array}{cc}
\wedge^{j} \Gamma^{\prime} & (-1)^{j} \beta_{j} \\
0 & \delta_{j}
\end{array}\right)
$$

We have to compute $\operatorname{ker} \varepsilon_{j}$.

Of course, the $j$-th syzygies of $J^{\prime}$ are in the kernel of $\varepsilon_{j}$ and so we can define a $\operatorname{map}\left(\wedge^{j+1} \Gamma^{\prime}, 0\right)^{t}: \wedge^{j+1} P\left(-\operatorname{deg} g_{1}\right) \rightarrow H_{j}$.

Claim 5. The $j$-th syzygies of $I$ can be extended to $j$-th syzygies of $\mathfrak{B}$.

To this end, we consider $x \in F_{j+1}$ and its image via $\left((-1)^{j+1} \beta_{j+1}, \delta_{j+1}\right)^{t}$ :

$$
\begin{gathered}
\varepsilon_{j}\left(\begin{array}{c}
(-1)^{j+1} \beta_{j+1}(x) \\
\delta_{j+1}(x)
\end{array}\right)=\left(\begin{array}{cc}
\wedge^{j} \Gamma^{\prime} & (-1)^{j} \beta_{j} \\
0 & \delta_{j}
\end{array}\right)\left(\begin{array}{c}
(-1)^{j+1} \beta_{j+1}(x) \\
\delta_{j+1}(x)
\end{array}\right) \\
=\left(\begin{array}{c}
(-1)^{j+1} \wedge^{j} \Gamma^{\prime} \circ \beta_{j+1}(x)+(-1)^{j} \beta_{j} \circ \delta_{j+1}(x) \\
\delta_{j} \circ \delta_{j+1}(x)
\end{array}\right) \\
=\left(\begin{array}{c}
(-1)^{j+1} \beta_{j} \circ \delta_{j+1}(x)+(-1)^{j} \beta_{j} \circ \delta_{j+1}(x) \\
0
\end{array}\right)=\left(\begin{array}{l}
0 \\
0
\end{array}\right) .
\end{gathered}
$$

Hence, we can define the map $\varepsilon_{j+1}: H_{j+1}=\wedge^{j+1} P\left(-\operatorname{deg} g_{1}\right) \oplus F_{j+1} \rightarrow H_{j}$ as

$$
\varepsilon_{j+1}=\left(\begin{array}{cc}
\wedge^{j+1} \Gamma^{\prime} & (-)^{j+1} \beta_{j+1} \\
0 & \delta_{j+1}
\end{array}\right)
$$


Claim 6. $\operatorname{Im} \varepsilon_{j+1}=\operatorname{ker} \varepsilon_{j}$.

Let $\left(y^{\prime}, y\right)^{t} \in \operatorname{ker} \varepsilon_{j}$. Of course, $\delta_{j}(y)=0$, and so there exists $x \in F_{j+1}$ such that $\delta_{j+1}(x)=y$. Then, $\left(y^{\prime}-(-1)^{j+1} \beta_{j+1}(x), 0\right)^{t} \in \operatorname{ker} \varepsilon_{j}$, and so there exists $x^{\prime} \in \wedge^{j+1} P\left(-\operatorname{deg} g_{1}\right)$ such that $\wedge^{j+1} \Gamma^{\prime}\left(x^{\prime}\right)=y^{\prime}-(-1)^{j+1} \beta_{j+1}(x)$. Hence, $\varepsilon_{j+1}\left(\left(x^{\prime}, x\right)^{t}\right)=\left(y^{\prime}, y\right)^{t}$, and we get the claim.

To compute explicitly a free resolution of $\mathfrak{A}$, we need the comparison maps

$$
\Phi_{1}: P\left(-t_{I}+t_{J}\right) \rightarrow P\left(-\operatorname{deg} g_{1}\right) \oplus \tilde{F}_{1} \oplus R\left(-t_{I}+t_{J}\right)
$$

and

$$
\Phi_{i}: \wedge^{i} P\left(-t_{I}+t_{J}\right) \rightarrow H_{i}=\wedge^{i} P\left(-\operatorname{deg} g_{i}\right) \oplus F_{i}, \quad i=2, \ldots, c-1,
$$

obtained by lifting the map $\psi$ in the exact sequence (6).

Remark 3.7. We define the maps $\bar{\beta}_{c-i}: \wedge^{i} P\left(t_{J}\right) \rightarrow F_{i}\left(t_{I}\right)$ as the composition of maps

$$
\left(F_{c-i}^{*} \stackrel{\sim}{\longrightarrow} F_{i}\left(t_{I}\right)\right) \circ \beta_{c-i}^{*} \circ\left(\theta_{i}: \wedge^{i} P\left(t_{J}\right) \stackrel{\sim}{\longrightarrow}\left(\wedge^{c-i-1} P\left(-\operatorname{deg} g_{1}\right)\right)^{*}\right) .
$$

From our choice for the minimal resolution of a Gorenstein ideal (see (3)), we know that the isomorphism on the left side is the identity map. Otherwise, for the characterization of complete intersection ideals, the isomorphism $\theta_{i}$ depends on the choice of bases for $\wedge^{i} P\left(t_{J}\right)$ and $\left(\wedge^{c-i-1} P\left(-\operatorname{deg} g_{1}\right)\right)^{*}$.

By dualizing the equation (2), we obtain

$$
\beta_{i}^{*} \circ\left(\wedge^{i-1} \Gamma^{\prime}\right)^{*}=\delta_{i}^{*} \circ \beta_{i-1}^{*} .
$$

By composing the left hand side with the isomorphisms $\theta_{i}$ we can write

$$
\beta_{i}^{*} \circ \theta_{c-i} \circ \theta_{c-i}^{-1} \circ\left(\wedge^{i-1} \Gamma^{\prime}\right)^{*} \circ \theta_{c-i+1}=\bar{\beta}_{i} \circ\left(\wedge^{c-i+1} \Gamma^{\prime}\left(t_{J}\right)\right),
$$

and hence we get

$$
\bar{\beta}_{i} \circ \wedge^{c-i+1} \Gamma\left(t_{J}\right)=\delta_{i}^{*} \circ \bar{\beta}_{i-1}=\delta_{c-i+1}\left(t_{I}\right) \circ \bar{\beta}_{i-1} .
$$

Before computing the comparison maps, we prove the existence of some maps with prescribed properties.

Lemma 3.8. There exist maps $\varphi_{i}: \wedge^{i} P\left(-t_{I}+t_{J}\right) \rightarrow \wedge^{i} P\left(-\operatorname{deg} g_{i}\right)$ such that

$$
\Gamma^{\prime} \circ \varphi_{1}=\beta_{1} \circ \bar{\beta}_{c-1}\left(-t_{I}\right)
$$

and

$$
\wedge^{i} \Gamma^{\prime} \circ \varphi_{i}=\varphi_{i-1} \circ \wedge^{i} \Gamma\left(-t_{I}+t_{J}\right)-(-1)^{i} \beta_{i} \circ \bar{\beta}_{c-i}\left(-t_{I}\right), \quad i=2, \ldots, c-1 .
$$

Proof. The existence of $\varphi_{1}$ stems from the inclusion

$$
\operatorname{Im}\left(\beta_{1} \circ \bar{\beta}_{c-1}\left(-t_{I}\right)\right) \subseteq \operatorname{Im}\left(\Gamma^{\prime}\right)=J^{\prime} .
$$

Because of the definition of $\bar{\beta}_{c-1}$, we have

$$
\operatorname{Im}\left(\beta_{1} \circ \bar{\beta}_{c-1}\left(-t_{I}\right)\right)=\operatorname{Im}\left(\beta_{1} \circ \beta_{c-1}^{*}\left(-t_{I}\right)\right) .
$$

If $\beta_{c-1}$ is represented by the matrix $B=\left(b_{i, j}\right)$, then, by (9), we have

$$
b_{i, 1} f_{1}+\cdots+b_{1, k} f_{k}+b_{i, k+1} g_{1}=f g_{i}, \quad i=2, \ldots, c .
$$


By Remark 3.1 $f_{i} \in J^{\prime}$, and so $b_{i, k+1} g_{1} \in J^{\prime}$, i.e., $b_{i, k+1} \in J^{\prime}: g_{1} R=J^{\prime}$, for $i=2, \ldots, c$. The map $\beta_{1} \circ \beta_{c-1}^{*}\left(-t_{I}\right)$ is represented by $\left(b_{2, k+1}, b_{3, k+1}, \ldots, b_{c, k+1}\right)$, and so the inclusion follows, and $\varphi_{1}$ exists.

Now, we go on by induction. Thus, we assume the existence of $\varphi_{i-1}$ and we prove the existence of $\varphi_{i}$ such that $\wedge^{i} \Gamma^{\prime} \circ \varphi_{i}=\varphi_{i-1} \circ \wedge^{i} \Gamma\left(-t_{I}+t_{J}\right)-(-1)^{i} \beta_{i} \circ \bar{\beta}_{c-i}\left(-t_{I}\right)$.

As before, we can define the map $\varphi_{i}$ if, and only if,

$$
\operatorname{Im}\left(\varphi_{i-1} \circ \wedge^{i} \Gamma\left(-t_{I}+t_{J}\right)-(-1)^{i} \beta_{i} \circ \bar{\beta}_{c-i}\left(-t_{I}\right)\right) \subseteq \operatorname{Im}\left(\wedge^{i} \Gamma^{\prime}\right)=\operatorname{ker}\left(\wedge^{i-1} \Gamma^{\prime}\right) .
$$

But, we have

$$
\begin{gathered}
\wedge^{i-1} \Gamma^{\prime} \circ\left(\varphi_{i-1} \circ \wedge^{i} \Gamma\left(-t_{I}+t_{J}\right)-(-1)^{i} \beta_{i} \circ \bar{\beta}_{c-i}\left(-t_{I}\right)\right) \\
=\wedge^{i-1} \Gamma^{\prime} \circ \varphi_{i-1} \circ \wedge^{i} \Gamma\left(-t_{I}+t_{J}\right)-(-1)^{i} \wedge^{i-1} \Gamma^{\prime} \circ \beta_{i} \circ \bar{\beta}_{c-i}\left(-t_{I}\right) \\
=(\text { by induction hypothesis }) \\
=\varphi_{i-2} \circ \wedge^{i-1} \Gamma\left(-t_{I}+t_{J}\right) \circ \wedge^{i} \Gamma\left(-t_{I}+t_{J}\right) \\
-(-1)^{i-1} \beta_{i-1} \circ \bar{\beta}_{c-i+1}\left(-t_{I}\right) \circ \wedge^{i} \Gamma\left(-t_{I}+t_{J}\right) \\
-(-1)^{i} \wedge^{i-1} \Gamma^{\prime} \circ \beta_{i} \circ \bar{\beta}_{c-i}\left(-t_{J}\right)=(\text { by (9) }) \\
=(-1)^{i} \beta_{i-1} \circ \bar{\beta}_{c-i+1}\left(-t_{I}\right) \circ \wedge^{i} \Gamma\left(-t_{I}+t_{J}\right)-(-1)^{i} \beta_{i-1} \circ \delta_{i} \circ \bar{\beta}_{c-i}\left(-t_{I}\right) \\
=(\text { by (12) })=0 .
\end{gathered}
$$

Hence, we can define the map $\varphi_{i}$ with the prescribed property.

Now, we compute the comparison maps.

Proposition 3.9. The comparison map $\Phi_{i}: \wedge^{i} P\left(-t_{I}+t_{J}\right) \rightarrow H_{i}$ can be defined as

$$
\Phi_{1}=\left(\begin{array}{c}
g \cdot i d+\varphi_{1} \\
p \circ \bar{\beta}_{c-1}\left(-t_{I}\right)
\end{array}\right), \quad \Phi_{i}=\left(\begin{array}{c}
g \cdot i d+\varphi_{i} \\
\bar{\beta}_{c-i}\left(-t_{I}\right)
\end{array}\right), \quad i=2, \ldots, c-1,
$$

where $p: F_{1} \rightarrow \tilde{F}_{1}$ is the projection.

Proof. It is enough to verify that

$$
\begin{aligned}
& \varepsilon_{1} \circ \Phi_{1}=\left(f+g_{1} g\right) \circ \Gamma\left(-t_{I}+t_{J}\right), \\
& \quad \varepsilon_{i} \circ \Phi_{i}=\Phi_{i-1} \circ \wedge^{i} \Gamma\left(-t_{I}+t_{J}\right), \quad i=2, \ldots, c-1 .
\end{aligned}
$$

We have

$$
\begin{gathered}
\varepsilon_{1} \circ \Phi_{1}=\left(g_{1} \Gamma^{\prime}, \delta_{1 \mid \tilde{F}_{1}}\right)\left(\begin{array}{c}
g \cdot i d+\varphi_{1} \\
p \circ \bar{\beta}_{c-1}\left(-t_{I}\right)
\end{array}\right) \\
=g g_{1} \Gamma\left(-t_{I}+t_{J}\right)+g_{1} \Gamma^{\prime} \circ \varphi_{1}+\delta_{1 \mid \tilde{F}_{1}} \circ p \circ \bar{\beta}_{c-1}\left(-t_{I}\right) \\
=\left(\text { by the construction of } \varphi_{1}\right) \\
=g_{1} g \Gamma\left(-t_{I}+t_{J}\right)+g_{1} \beta_{1} \circ \bar{\beta}_{c-1}\left(t_{I}\right)+\delta_{1 \mid \tilde{F}_{1}} \circ p \circ \bar{\beta}_{c-1}\left(-t_{I}\right) \\
=\left(\text { by definition of } \delta_{1 \mid \tilde{F}_{1}}\right) \\
=g_{1} g \Gamma\left(-t_{I}+t_{J}\right)+\delta_{1} \circ \bar{\beta}_{c-1}\left(-t_{I}\right)=(\text { by (12) }) \\
=g_{1} g \Gamma\left(-t_{I}+t_{J}\right)+\bar{\beta}_{c}\left(-t_{I}\right) \circ \Gamma\left(-t_{I}+t_{J}\right) \\
=\left(\text { by definition of } \beta_{c}\right)=\left(f+g_{1} g\right) \circ \Gamma\left(-t_{I}+t_{J}\right),
\end{gathered}
$$

and so we get the relation (13). 
Now, we prove the equation (14). At first, we assume $i \geq 3$. We have

$$
\begin{aligned}
\varepsilon_{i} \circ \Phi_{i} & =\left(\begin{array}{cc}
\wedge^{i} \Gamma^{\prime} & (-1)^{i} \beta_{i} \\
0 & \delta_{i}
\end{array}\right)\left(\begin{array}{c}
g \\
\bar{\beta}_{c-i}\left(-t_{I}\right)
\end{array}\right) \\
& =\left(\begin{array}{c}
g \cdot \wedge^{i} \Gamma^{\prime}+\wedge^{i} \Gamma^{\prime} \circ \varphi_{i}+(-1)^{i} \beta_{i} \circ \bar{\beta}_{c-i}\left(-t_{I}\right) \\
\delta_{i} \circ \bar{\beta}_{c-i}\left(t_{I}\right)
\end{array}\right) \\
& =\left(\begin{array}{c}
g \cdot \wedge^{i} \Gamma^{\prime}+\varphi_{i-1} \circ \wedge^{i} \Gamma\left(t_{J}-t_{I}\right)-(-1)^{i} \beta_{i} \circ \bar{\beta}_{c-i}\left(-t_{I}\right) \\
+(-1)^{i} \beta_{i} \circ \bar{\beta}_{c-i}\left(-t_{I}\right) \\
\bar{\beta}_{c-i+1}\left(-t_{I}\right) \circ \wedge^{i} \Gamma\left(-t_{I}+t_{J}\right)
\end{array}\right) \\
& =\left(\begin{array}{c}
g \\
\bar{\beta}_{c-i+1}\left(-t_{I}\right)
\end{array}\right) \circ \wedge^{i} \Gamma\left(-t_{I}+t_{J}\right),
\end{aligned}
$$

and so the relation (14) holds true for $i \geq 3$.

If $i=2$, we have

$$
\begin{aligned}
\varepsilon_{2} \circ \Phi_{2} & =\left(\begin{array}{cc}
\wedge^{2} \Gamma^{\prime} & \beta_{2} \\
0 & \tilde{\delta}_{2}
\end{array}\right)\left(\begin{array}{c}
g \cdot i d+\varphi_{2} \\
\bar{\beta}_{c-2}\left(-t_{I}\right)
\end{array}\right) \\
& =\left(\begin{array}{c}
g \cdot \wedge^{2} \Gamma^{\prime}+\wedge^{2} \Gamma^{\prime} \circ \varphi_{2}+\beta_{2} \circ \bar{\beta}_{c-2}\left(-t_{I}\right) \\
\tilde{\delta}_{2} \circ \bar{\beta}_{c-2}\left(-t_{I}\right)
\end{array}\right) \\
& =\left(\begin{array}{c}
g \cdot \wedge^{2} \Gamma^{\prime}+\varphi_{1} \circ \wedge^{2} \Gamma\left(-t_{I}+t_{J}\right)-\beta_{2} \circ \bar{\beta}_{c-2}\left(-t_{I}\right)+\beta_{2} \circ \bar{\beta}_{c-2}\left(-t_{I}\right) \\
p \circ \delta_{2} \circ \bar{\beta}_{c-2}\left(-t_{I}\right)
\end{array}\right) \\
& =\left(\begin{array}{c}
g \wedge^{2} \Gamma^{\prime}+\varphi_{1} \circ \wedge^{2} \Gamma\left(-t_{I}+t_{J}\right) \\
p \circ \bar{\beta}_{c-1}\left(-t_{I}\right) \circ \wedge^{2} \Gamma\left(-t_{I}+t_{J}\right)
\end{array}\right)=\Phi_{1} \circ \wedge^{2} \Gamma\left(-t_{I}+t_{J}\right),
\end{aligned}
$$

and hence the claim follows.

Proof of Theorem 3.2. Using the free resolution of $\mathfrak{B}$ computed in Lemma 3.6, the exact sequence (6), and the comparison maps computed in Proposition 3.9, we can compute a free resolution of $\mathfrak{A}$, by a mapping cone. In particular, the last map in the resolution of $\mathfrak{A}$ is

$$
\begin{array}{ccc}
\wedge^{c-1} P\left(-t_{I}+t_{J}\right) & \stackrel{\wedge^{c-1} \Gamma\left(-t_{I}+t_{J}\right)}{\longrightarrow} & \wedge^{c-2} P\left(-t_{I}+t_{J}\right) \\
\oplus & \searrow(-1)^{c} \Phi_{c-1} & \oplus \\
H_{c} & \overrightarrow{\varepsilon_{c}} & H_{c-1}
\end{array}
$$

Of course, the following equalities hold:

$$
\begin{gathered}
\wedge^{c-1} P\left(-t_{I}+t_{J}\right) \cong R\left(-t_{I}+t_{J}-t_{J}^{\prime}\right)=R\left(-t_{I}+\operatorname{deg} g_{1}\right), \\
H_{c}=\wedge^{c} P\left(-\operatorname{deg} g_{1}\right) \oplus F_{c}=F_{c}=R\left(-t_{I}\right), \\
\Phi_{c-1}=\left(\begin{array}{c}
g \cdot I d_{\wedge^{c-1} P}+\varphi_{c-1} \\
\bar{\beta}_{1}\left(-t_{I}\right)
\end{array}\right) .
\end{gathered}
$$

The map $\bar{\beta}_{1}: R\left(\operatorname{deg} g_{1}\right) \rightarrow R\left(\operatorname{deg} g_{1}\right) \oplus \tilde{F}_{1}^{*}$ is the identity onto the first addendum, by Remark 3.5, and so the last free module in the resolution of $\mathfrak{A}$ has rank 1 .

Now, we want to prove that $\mathfrak{A}$ is a codimension $c$ ideal. The minimal free resolution of $\mathfrak{A}$ has length at most $c$, and so the codimension of $\mathfrak{A}$ cannot be greater than $c$. 
Let $\mathfrak{P}$ be a minimal prime ideal associated to $\mathfrak{A}$, of the same codimension. Then,

$$
\left(g_{1} g_{2}, \ldots, g_{1} g_{c}, f_{1}, \ldots, f_{k}, f+g_{1} g\right) \subseteq \mathfrak{P}
$$

If $g_{1} \in \mathfrak{P}$, then $\left(g_{1}, f_{1}, \ldots, f_{k}, f\right) \subseteq \mathfrak{P}$, and $\operatorname{so} \operatorname{codim}(\mathfrak{P}) \geq c$.

If $g_{1} \notin \mathfrak{P}$, then $\left(g_{2}, \ldots, g_{c}, f+g_{1} g\right) \subseteq \mathfrak{P}$, and again we have $\operatorname{codim}(\mathfrak{P}) \geq c$.

But $\operatorname{codim} A=\operatorname{codim} \mathfrak{P} \geq c$ and $\operatorname{codim} \mathfrak{A} \leq \operatorname{pd}(\mathfrak{A})=c$. Hence, $\mathfrak{A}$ has codimension $c$, and it is a Gorenstein ideal of codimension $c$.

Remark 3.10. From the computation of the resolution of $\mathfrak{A}$ we get that the last free module is $R\left(-t_{I}\right)$, and so $\mathfrak{A}$ and $I$ have the same free module at the last place in their minimal free resolutions.

Remark 3.11. The ideal $\mathfrak{A}$ is generated by $\nu(I)+c-1$ generators, where $\nu(I)=k+1$ is the minimal number of generators of $I$. It may happen that some of them are not minimal. Then, we can only say that $\mathfrak{A}$ is minimally generated by $\nu(\mathfrak{A})$ forms, where $c \leq \nu(\mathfrak{A}) \leq \nu(I)+c-1$. In some cases, it may happen that $\nu(\mathfrak{A})<\nu(I)$ (see, for example Proposition 3.17 below).

Now, we want to relate the Hilbert functions of $I, J, J^{\prime}, I_{\text {res }}, \mathfrak{A}$ and $J^{\prime \prime}=$ $\left(g_{2}, \ldots, g_{c}, f+g_{1} g\right)$.

Proposition 3.12. Let $I, J, J^{\prime}, J^{\prime \prime}, I_{\mathrm{res}}, \mathfrak{A}$ be as above. Then:

(1) $h_{R / \mathfrak{A}}(z)=h_{R / I}(z)+h_{R / J^{\prime}}\left(z-\operatorname{deg} g_{1}\right)-h_{R / J^{\prime}}\left(z-t_{I}+t_{J}\right)$;

(2) $h_{R / \mathfrak{A}}(z)=h_{R / I}(z)+h_{R / J^{\prime \prime}}(z)-h_{R / J}(z)$;

(3) $h_{R / \mathfrak{A}}(z)=h_{R / I_{\text {res }}}(z)+h_{R / J^{\prime}}\left(z-\operatorname{deg} g_{1}\right)-h_{R / J^{\prime}}\left(z-t_{I}+t_{J}-\operatorname{deg} g_{1}\right)$.

Proof. From the exact sequence

$$
\begin{aligned}
& \wedge^{c-1} P\left(-\operatorname{deg} g_{1}\right)
\end{aligned}
$$

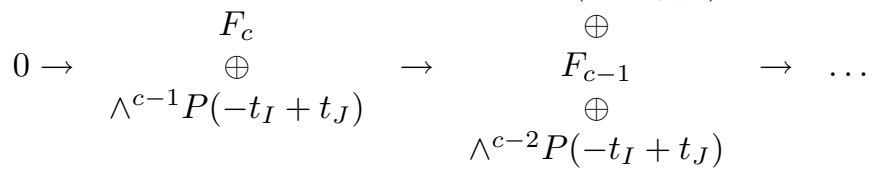

$$
\begin{aligned}
& \wedge^{2} P\left(-\operatorname{deg} g_{1}\right) \quad P\left(-\operatorname{deg} g_{1}\right) \\
& \cdots \rightarrow \begin{array}{ccccc}
\oplus & & \oplus & & R \\
F_{2} & \rightarrow & F_{1} \\
\oplus & & \oplus & \oplus \\
P\left(-t_{I}+t_{J}\right) & & & \rightarrow\left(-t_{I}+t_{J}\right)
\end{array} \quad \rightarrow R / \mathfrak{A} \rightarrow 0
\end{aligned}
$$

we obtain

$$
\begin{aligned}
h_{R / \mathfrak{A}}(z) & =h_{R / I}(z)+\left[\sum_{i=0}^{c-1} \operatorname{dim}\left[\wedge^{i} P\left(-\operatorname{deg} g_{1}\right)\right]_{z}\right]-\left[\sum_{i=0}^{c-1} \operatorname{dim}\left[\wedge^{i} P\left(-t_{I}+t_{J}\right)\right]_{z}\right] \\
& =h_{R / I}(z)+h_{R / J^{\prime}}\left(z-\operatorname{deg} g_{1}\right)-h_{R / J^{\prime}}\left(z-t_{I}+t_{J}\right) \\
& =h_{R / I}(z)+h_{R / J^{\prime}}\left(z-\operatorname{deg} g_{1}\right)-h_{R / J^{\prime}}(z)+h_{R / J^{\prime}}(z)-h_{R / J^{\prime}}\left(z-t_{I}+t_{J}\right) \\
& =h_{R / I}(z)-h_{R / J}(z)+h_{R / J^{\prime \prime}}(z) ;
\end{aligned}
$$


then (1) and (2) follow. For (3) it is enough to remember the relation between the $h$-vectors of $I_{\text {res }}, J$ and $I$ ([14, Corollary 5.2.19), and so we have

$$
\begin{gathered}
h_{R / \mathfrak{A}}(z)-h_{R / I_{\mathrm{res}}}(z)=h_{R / \mathfrak{A}}(z)-\left[h_{R / I}\left(t_{I}-c-z\right)-h_{R / J}\left(t_{I}-c-z\right)\right] \\
=h_{R / \mathfrak{A}}(z)-\left[h_{R / I}(z)-h_{R / J}\left(t_{J}-c-\left(t_{J}-t_{I}+z\right)\right)\right] \\
=h_{R / \mathfrak{A}}(z)-\left[h_{R / I}(z)-h_{R / J}\left(t_{J}-t_{I}+z\right)\right] \\
=h_{R / \mathfrak{A}}(z)-h_{R / J}(z)+h_{R / J^{\prime \prime}}(z)-h_{R / I}(z)+h_{R / J}\left(t_{J}-t_{I}+z\right) \\
=h_{R / J^{\prime}}\left(z-\operatorname{deg} g_{1}\right)-h_{R / J^{\prime}}\left(z-t_{I}+t_{J}-\operatorname{deg} g_{1}\right) .
\end{gathered}
$$

From the previous relations on the Hilbert functions, we deduce a relation about the degrees of the corresponding rings.

Corollary 3.13. Let $I, J, J^{\prime \prime}, \mathfrak{A}$ be as above. Then,

$$
\operatorname{deg}(R / \mathfrak{A})=\operatorname{deg}(R / I)+\left(t_{I}-t_{J}-\operatorname{deg} g_{1}\right) \prod_{i=2}^{c} \operatorname{deg} g_{i} .
$$

Proof. The rings $R / I, R / J, R / J^{\prime \prime}$, and $R / \mathfrak{A}$ have the same dimension, and so, looking at the leading coefficients of their Hilbert polynomials, from Proposition 3.12 2) we get

$$
\operatorname{deg}(R / \mathfrak{A})=\operatorname{deg}(R / I)-\operatorname{deg}(R / J)+\operatorname{deg}\left(R / J^{\prime \prime}\right) .
$$

The ideals $J$ and $J^{\prime \prime}$ are complete intersection ideals, and so their degrees are

$$
\operatorname{deg}(R / J)=\prod_{i=1}^{c} \operatorname{deg} g_{i} \quad \operatorname{deg}\left(R / J^{\prime \prime}\right)=\left(t_{I}-t_{J}\right) \prod_{i=2}^{c} \operatorname{deg} g_{i} .
$$

By substituting in the previous relation we get the claim.

Remark 3.14. From Proposition $\underline{3.12} 1$ ), it follows that if $\operatorname{deg} g_{1}<t_{I}-t_{J}$, then $h_{R / \mathfrak{A}}(z) \geq h_{R / I}(z)$, for each $z \in \mathbb{Z}$.

In fact, the Hilbert function $h_{R / J^{\prime}}(z)$ is non-decreasing, because $\operatorname{dim}\left(R / J^{\prime}\right) \geq 1$, and so, under our hypothesis, $h_{R / J^{\prime}}\left(z-\operatorname{deg} g_{1}\right) \geq h_{R / J^{\prime}}\left(z-t_{I}+t_{J}\right)$.

Of course, if $\operatorname{deg} g_{1}>t_{I}-t_{J}$, then the reverse inequality holds.

Now, we show that $I_{\text {res }}$ and $J^{\prime \prime}$ are linked by the Gorenstein ideals $\mathfrak{A}$.

Proposition 3.15. $\mathfrak{A}: I_{\mathrm{res}}=\left(g_{2}, \ldots, g_{c}, f+g_{1} g\right)=J^{\prime \prime}$.

Proof. Obviously, $J^{\prime \prime} \subseteq \mathfrak{A}: I_{\text {res }}$. On the other hand,

$$
\begin{gathered}
h_{R / \mathfrak{A}: I_{\mathrm{res}}}(z)=h_{R / \mathfrak{A}}\left(t_{I}-c-z\right)-h_{R / I_{\mathrm{res}}}\left(t_{I}-c-z\right) \\
=h_{R / \mathfrak{A}}(z)-\left[h_{R / I}\left(t_{I}-c-t_{I}+c+z\right)-h_{R / J}\left(t_{I}-c-t_{I}+c+z\right)\right] \\
=h_{R / J}(z)+h_{R / J^{\prime \prime}}(z)-h_{R / J}(z)=h_{R / J^{\prime \prime}}(z),
\end{gathered}
$$

and so the equality follows from the equality of their Hilbert functions.

Remark 3.16. If the link is geometric, then a stronger relation holds:

$$
I_{\text {res }} \cap J^{\prime \prime}=\mathfrak{A} \text {. }
$$

Now, we study what happens if we reverse the construction of Theorem 3.2 In particular, we have the following. 
Proposition 3.17. Let $I, J, J^{\prime \prime}, \mathfrak{A}$ be as above. Then, $\mathfrak{A} \subset J^{\prime \prime}, \mathfrak{A}: J^{\prime \prime}=\mathfrak{A}+g_{1} R$, and the ideal constructed as in Theorem 3.2 using the complete intersection ideal $J$ is the (Gorenstein) ideal I.

Proof. The inclusion $\mathfrak{A} \subset J^{\prime \prime}$ is trivial. By Proposition 3.15 it follows that $\mathfrak{A}$ : $J^{\prime \prime}=I_{\text {res }}$. Then the equality $\mathfrak{A}: J^{\prime \prime}=\mathfrak{A}+g_{1} R$ follows by $I_{\text {res }}=\mathfrak{A}+g_{1} R$, which is trivial. At last, if we apply the construction of Theorem [3.2 to $\mathfrak{A} \subset J^{\prime \prime}$ using as new complete intersection the ideal $J$, we immediately get the ideal $I$, because $f g_{i} \in I$ for every $i=2, \ldots, c$ (compare with the proof of Lemma 3.3).

Of course, this proposition shows that the number of generators of the new Gorenstein ideal obtained from the construction of Theorem 3.2 can decrease with respect to the number of generators of the starting Gorenstein ideal (compare with Remark 3.11).

To conclude this section, we want to give an example of Gorenstein ideals arising from Theorem 3.2 .

Example 3.18. As an example, we show that every reduced zero-dimensional arithmetically Gorenstein scheme of degree $n+2$ in $\mathbb{P}^{n}$ arises from the construction described in Theorem 3.2 .

Let $X=\left\{P_{1}, \ldots, P_{n+2}\right\} \subset \mathbb{P}^{n}$ be such a scheme, with $P_{i} \neq P_{j}$ for $i \neq j$, and let $\mathfrak{A} \subset R$ be the defining ideal of $X$. It is known that $X$ is an arithmetically Gorenstein scheme if, and only if, no $n+1$ of them are contained in a hyperplane ( 8 , Theorem $5)$.

Let $r$ be the line through $P_{n+1}, P_{n+2}$ whose ideal is $J^{\prime}=\left(g_{2}, \ldots, g_{n}\right)$, where $g_{2}, \ldots, g_{n}$ are, of course, linear forms. Let $H=V\left(g_{1}\right)$ be the hyperplane containing $P_{1}, \ldots, P_{n}$. Then $r \cap H=\{A\}$ is a point not in $X$ defined by the ideal $J=$ $\left(g_{1}, \ldots, g_{n}\right)$. We set $Y=\left\{A, P_{1}, \ldots, P_{n}\right\} \subset H$, and we denote by $I \subset R$ its defining ideal. We can choose $I$ generated by $\left(g_{1}, f_{1}, \ldots, f_{k}\right)$.

Claim. $Y$ is an arithmetically Gorenstein scheme.

The claim is equivalent to showing that no subset of $Y$ of degree $n$ is contained in a codimension 2 linear subspace of $\mathbb{P}^{n}$.

By contradiction, if $\left\{A, P_{1}, \ldots, P_{n-1}\right\} \subset W$ is a linear subspace of codimension 2 , then $\left\{A, P_{1}, \ldots, P_{n-1}, P_{n+1}, P_{n+2}\right\} \subset W^{\prime}$, where $W^{\prime}$ is the linear subspace spanned by $W$ and $r . W^{\prime}$ is a codimension 1 linear subspace by the Grassmann formula, and so we get that $X$ is not arithmetically Gorenstein. The contradiction proves the claim.

The point $\{A\}$ is a complete intersection scheme contained in $Y$, and the residual scheme is $\left\{P_{1}, \ldots, P_{n}\right\} \subset X$ defined by the ideal $I_{\text {res }}=I: J$. The minimal free resolutions of $I$ and $J$ have the following shapes:

$$
\begin{aligned}
0 \rightarrow R(-n-2) \rightarrow F_{1}^{*}(-n-2) & \rightarrow \cdots \rightarrow F_{1} \rightarrow I \rightarrow 0, \\
& 0 \rightarrow R(-n) \rightarrow \wedge^{n-1} G \rightarrow \cdots \rightarrow G \rightarrow J \rightarrow 0,
\end{aligned}
$$

and so $I_{\text {res }}=I+f R$, where $\operatorname{deg} f=2$.

Now, we consider the ideal generated by $f+g_{1} g, g_{2}, \ldots, g_{n}$, where $g$ is a linear form. If we choose $g \notin J^{\prime}$ in such a way that $f\left(P_{i}\right)+h\left(P_{i}\right) g\left(P_{i}\right)=0$ for $i=$ $n+1, n+2$, we have that $J^{\prime}+\left(f+g_{1} g\right) R=I_{P_{n+1}, P_{n+2}}$, and it is a complete intersection ideal. Hence, $\left(J^{\prime}+\left(f+g_{1} g\right) R\right) \cap I_{\text {res }}=\mathfrak{A}$, and $X$ can be obtained from Theorem 3.2 . 


\section{The Codimension 3 CASE}

In this section, we want to relate the Buchsbaum-Eisenbud matrices of the Gorenstein ideals $I$ and $\mathfrak{A}$, in the Pfaffian case.

To obtain the result we want, first we rewrite diagram (3) in the codimension 3 case, and so we have

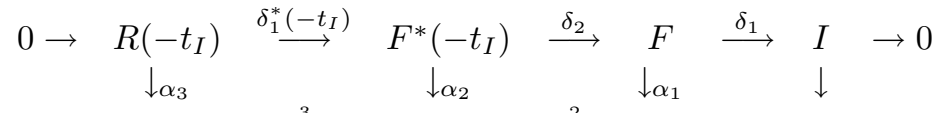

$$
\begin{aligned}
& 0 \rightarrow \quad \wedge^{3} G \quad \stackrel{\wedge^{3} \gamma}{\longrightarrow} \quad \wedge^{2} G \quad \stackrel{\wedge^{2} \gamma}{\longrightarrow} G \stackrel{\gamma}{\longrightarrow} J \rightarrow 0 .
\end{aligned}
$$

Now, we compute matrices representing the maps $\alpha_{1}, \alpha_{2}, \alpha_{3}$ in terms of the matrices representing the other maps. First, we state some notation.

Notation. We set $g_{1}, f_{1}, \ldots, f_{m}$ a minimal set of generators of $I$, and $g_{1}, g_{2}, g_{3}$ a minimal set of generators of $J$. Because the codimension is $3, m$ is even ([22, theorem on p. 229). Then, it is straightforward that

$$
F=R\left(-\operatorname{deg} g_{1}\right) \oplus\left(\bigoplus_{i=1}^{m} R\left(-\operatorname{deg} f_{i}\right)\right) \quad \text { and } \quad G=\bigoplus_{i=1}^{3} R\left(-\operatorname{deg} g_{i}\right)
$$

and the maps $\delta_{1}$ and $\gamma$ are represented by

$$
D_{1}=\left(g_{1}, f_{1}, \ldots, f_{m}\right) \quad \text { and } \quad C_{1}=\left(g_{1}, g_{2}, g_{3}\right)
$$

If $I$ is a Pfaffian ideal, and if we choose suitable bases for $F$ and $F^{*}\left(-t_{I}\right)$, the map $\delta_{2}$ is represented by a skew-symmetric matrix

$$
D_{2}=\left(d_{i j}\right)_{0 \leq i, j \leq m},
$$

while the last map $\delta_{1}^{*}\left(-t_{I}\right)$ is then represented by the matrix

$$
D_{3}=D_{1}^{t} \text {. }
$$

If we choose the canonical basis $\left\{e_{1}, e_{2}, e_{3}\right\}$ for the module $G,\left\{e_{2} \wedge e_{3}, e_{3} \wedge e_{1}, e_{1} \wedge\right.$ $\left.e_{2}\right\}$ as basis of $\wedge^{2} G$, and $\left\{e_{1} \wedge e_{2} \wedge e_{3}\right\}$ as basis of $\wedge^{3} G$, then the maps $\wedge^{2} \gamma$ and $\wedge^{3} \gamma$ are represented by the matrices

$$
C_{2}=\left(\begin{array}{ccc}
0 & -g_{3} & g_{2} \\
g_{3} & 0 & -g_{1} \\
-g_{2} & g_{1} & 0
\end{array}\right) \quad \text { and } \quad C_{3}=C_{1}^{t}
$$

Now, we prove a lemma in which we relate the entries of the various matrices.

Lemma 4.1. With the above notation, we have:

(1) There exist $a_{2 j}, a_{3 j} \in R$ such that

$$
f_{j}=a_{2 j} g_{2}+a_{3 j} g_{3} \quad \text { for } j=1, \ldots, m .
$$

(2) There exist $b_{2 j}, b_{3 j} \in R$ such that

$$
d_{0 j}=b_{2 j} g_{2}+b_{3 j} g_{3} \quad \text { for } j=1, \ldots, m .
$$

(3) There exist $\lambda_{j} \in R$ such that

$$
b_{2 j} g_{1}+\sum_{l=1}^{m} a_{2 l} d_{l j}=\lambda_{j} g_{3} \quad \text { for } j=1, \ldots, m,
$$


and

$$
b_{3 j} g_{1}+\sum_{l=1}^{m} a_{3 l} d_{l j}=-\lambda_{j} g_{2} \quad \text { for } j=1, \ldots, m .
$$

(4) There exists $c \in R$ such that

$$
\sum_{l=1}^{m} a_{2 l} d_{l 0}=c g_{3}, \quad \sum_{l=1}^{m} a_{3 l} d_{l 0}=-c g_{2} .
$$

(5) There exists $c^{\prime} \in R$ such that

$$
c+\sum_{l=1}^{m} a_{2 l} b_{3 l}=c^{\prime} g_{2}, \quad \sum_{l=1}^{m} a_{2 l} b_{2 l}=-c^{\prime} g_{3} .
$$

(6) There exists $c^{\prime \prime} \in R$ such that

$$
c-\sum_{l=1}^{m} a_{3 l} b_{2 l}=c^{\prime \prime} g_{3}, \quad \sum_{l=1}^{m} a_{3 l} b_{3 l}=c^{\prime \prime} g_{2} .
$$

Proof. The first equality follows from Remark 3.1.

From $D_{1} D_{2}=0$ we get

$$
g_{1} d_{0 j}+\sum_{i=1}^{m} f_{i} d_{i j}=0 \quad \text { for } j=1, \ldots, m .
$$

Again from Remark 3.1, $f_{i} \in J^{\prime}$, and so $d_{0 j} \in J^{\prime}:\left(g_{1}\right) R=J^{\prime}$. Then, there exist $b_{2 j}, b_{3 j} \in R$ such that

$$
d_{0 j}=b_{2 j} g_{2}+b_{3 j} g_{3} \quad \text { for } j=1, \ldots, m,
$$

and so we have the second equality.

By substituting the first two equations in (22), we have

$$
g_{1}\left(b_{2 j} g_{2}+b_{3 j} g_{3}\right)+\sum_{i=1}^{m}\left(a_{2 i} g_{2}+a_{3 i} g_{3}\right) d_{i j}=0
$$

and hence

$$
g_{2}\left(b_{2 j} g_{1}+\sum_{i=1}^{m} a_{2 i} d_{i j}\right)+g_{3}\left(b_{3 j} g_{1}+\sum_{i=1}^{m} a_{3 i} d_{i j}\right)=0 .
$$

The sequence $g_{2}, g_{3}$ is regular, and so there exists $\lambda_{j} \in R$ such that

$$
b_{2 j} g_{1}+\sum_{i=1}^{m} a_{2 i} d_{i j}=\lambda_{j} g_{3}, \quad b_{3 j} g_{1}+\sum_{i=1}^{m} a_{3 i} d_{i j}=-\lambda_{j} g_{2}
$$

for each $j=1, \ldots, m$, and then the third equality follows.

Again from $D_{1} D_{2}=0$ we get

$$
\sum_{i=1}^{m} f_{i} d_{i 0}=0
$$

By substituting the first equation, we get

$$
g_{2} \sum_{l=1}^{m} a_{2 l} d_{l 0}+g_{3} \sum_{l=1}^{m} a_{3 l} d_{l 0}=0
$$


and so there exists $c \in R$ such that

$$
\sum_{l=1}^{m} a_{2 l} d_{l 0}=c g_{3}, \quad \sum_{l=1}^{m} a_{3 l} d_{l 0}=-c g_{2} .
$$

Because of the skew-symmetry of $D_{2}$, and the equations (24), (23), we get

$$
c g_{3}=-\sum_{l=1}^{m} a_{2 l}\left(b_{2 l} g_{2}+b_{3 l} g_{3}\right)
$$

and

$$
-c g_{2}=-\sum_{l=1}^{m} a_{3 l}\left(b_{2 l} g_{2}+b_{3 l} g_{3}\right) .
$$

These two equations give syzygies of $\left(g_{2}, g_{3}\right)$, and so we have also the last two equations.

Remark 4.2. The previous equalities don' t have unique solutions, in general. However, from now on in this section, we choose a solution for every equation, and we work with that one.

Now, we can compute matrices which represent the maps $\alpha_{i}$. Of course, the maps $\alpha_{i}$ are chosen once for ever and are unique up to homotopy.

Proposition 4.3. The map $\alpha_{1}$ is represented by

$$
A_{1}=\left(\begin{array}{cccc}
1 & 0 & \ldots & 0 \\
0 & a_{21} & \ldots & a_{2 m} \\
0 & a_{31} & \ldots & a_{3 m}
\end{array}\right),
$$

$\alpha_{2}$ is represented by

$$
A_{2}=\left(\begin{array}{cccc}
c & \lambda_{1} & \ldots & \lambda_{m} \\
0 & -b_{31} & \ldots & -b_{3 m} \\
0 & b_{21} & \ldots & b_{2 m}
\end{array}\right),
$$

and $\alpha_{3}$ is represented by

$$
A_{3}=\left(c-c^{\prime} g_{2}-c^{\prime \prime} g_{3}\right) .
$$

Proof. The map $\alpha_{1}$ is easily computed, and so $A_{1}$ is obvious using Lemma 4.1(1).

To compute the matrix $A_{2}$ we use the commutativity of the diagram (21), which gives $C_{2} A_{2}=A_{1} D_{2}$. If $\mathbf{a}_{j}$ is the $j$-th column of $A_{2}$, the previous relation on matrices can be written as

$$
C_{2} \mathbf{a}_{0}=\left(\begin{array}{c}
0 \\
\sum_{l=1}^{m} a_{2 l} d_{l 0} \\
\sum_{l=0}^{m} a_{3 l} d_{l 0}
\end{array}\right)
$$

and

$$
C_{2} \mathbf{a}_{j}=\left(\begin{array}{c}
d_{0 j} \\
\sum_{l=1}^{m} a_{2 l} d_{l j} \\
\sum_{l=1}^{m} a_{3 l} d_{l j}
\end{array}\right) .
$$

By Lemma 4.12),(3), and (4), we can write

$$
\left(\begin{array}{c}
0 \\
\sum_{l=1}^{m} a_{2 l} d_{l 0} \\
\sum_{l=0}^{m} a_{3 l} d_{l 0}
\end{array}\right)=C_{2}\left(\begin{array}{l}
c \\
0 \\
0
\end{array}\right)
$$


and

$$
\left(\begin{array}{c}
d_{0 j} \\
\sum_{l=1}^{m} a_{2 l} d_{l j} \\
\sum_{l=1}^{m} a_{3 l} d_{l j}
\end{array}\right)=C_{2}\left(\begin{array}{c}
\lambda_{j} \\
-b_{3 j} \\
b_{2 j}
\end{array}\right) .
$$

Hence, a possible choice for $A_{2}$ is

$$
A_{2}=\left(\begin{array}{cccc}
c & \lambda_{1} & \ldots & \lambda_{m} \\
0 & -b_{31} & \ldots & -b_{3 m} \\
0 & b_{21} & \ldots & b_{2 m}
\end{array}\right) .
$$

To compute $A_{3}$ we have to lift the matrix $A_{2}$. From the diagram (21), we have that $C_{3} A_{3}=A_{2} D_{3}$, i.e.

$$
\left(\begin{array}{l}
f g_{1} \\
f g_{2} \\
f g_{3}
\end{array}\right)=\left(\begin{array}{c}
c g_{1}+\sum_{j=1}^{m} \lambda_{j} f_{j} \\
-\sum_{j=1}^{m} b_{3 j} f_{j} \\
\sum_{j=1}^{m} b_{2 j} f_{j}
\end{array}\right) .
$$

We have the following equalities:

$$
\begin{gathered}
\sum_{j=1}^{m} b_{2 j} f_{j}=\text { by Lemma 4.1 }(1) \\
=\sum_{j=1}^{m} b_{2 j}\left(a_{2 j} g_{2}+a_{3 j} g_{3}\right)=g_{2} \sum_{j=1}^{m} a_{2 j} b_{2 j}+g_{3} \sum_{j=1}^{m} a_{3 j} b_{2 j}=\text { by Lemma 4.1](5),(6) } \\
=-c^{\prime} g_{2} g_{3}+\left(c-c^{\prime \prime} g_{3}\right) g_{3}=g_{3}\left(c-c^{\prime} g_{2}-c^{\prime \prime} g_{3}\right) .
\end{gathered}
$$

Hence, $f=c-c^{\prime} g_{2}-c^{\prime \prime} g_{3}$ gives the only element of $A_{3}$.

To apply the results of Section 3, we have to compute the maps $\beta_{1}, \beta_{2}, \beta_{3}$ in our case.

Corollary 4.4. The maps $\beta_{1}, \beta_{2}, \beta_{3}$ are represented respectively by the matrices

$$
\begin{gathered}
B_{1}=\left(\begin{array}{cccc}
1 & 0 & \ldots & 0
\end{array}\right), \\
B_{2}=\left(\begin{array}{llll}
0 & b_{21} & \ldots & b_{2 m} \\
0 & b_{31} & \ldots & b_{3 m}
\end{array}\right),
\end{gathered}
$$

and

$$
B_{3}=\left(c-c^{\prime} g_{2}-c^{\prime \prime} g_{3}\right) .
$$

Proof. By its definition (cf. Remark 3.5), $\beta_{h}=\pi_{h} \circ \alpha_{h}$, and so we have to compute the projections $\pi_{h}$.

$\pi_{1}: G \rightarrow R\left(-\operatorname{deg} g_{1}\right)$ is defined by setting $\pi_{1}\left(e_{j}\right)=\delta_{1 j}$, where $\delta_{i j}$ is the Kronecker symbol, and so the associated matrix is $\left(\begin{array}{lll}1 & 0 & 0\end{array}\right)$.

The map $\pi_{2}: \wedge^{2} G \rightarrow P$ is defined as $\pi_{2}\left(a_{1} e_{2} \wedge e_{3}+a_{2} e_{3} \wedge e_{1}+a_{3} e_{1} \wedge e_{2}\right)=$ $a_{3} e_{2}-a_{2} e_{3}$, and so the associated matrix is

$$
\left(\begin{array}{ccc}
0 & 0 & 1 \\
0 & -1 & 0
\end{array}\right)
$$

The map $\pi_{3}: \wedge^{3} G \rightarrow \wedge^{2} P$ is defined as $\pi_{3}\left(a e_{1} \wedge e_{2} \wedge e_{3}\right)=a e_{2} \wedge e_{3}$, and so the associated matrix is (1).

The result follows by multiplying the corresponding matrices. 
Now, we can show that $\mathfrak{A}$ is a Pfaffian ideal.

Theorem 4.5. Let I be a codimension 3 Gorenstein ideal generated by the Pfaffians of the skew-symmetric matrix $D_{2}$, and let $J$ be a codimension 3 complete intersection ideal containing I generated by the entries of $C_{1}$. Let $\tilde{D}_{2}$ be the submatrix of $D_{2}$ obtained by erasing the first row and the first column of $D_{2}$. Furthermore, let $\tilde{B}_{2}$ be equal to

$$
\tilde{B}_{2}=\left(\begin{array}{c}
0 \\
B_{2}
\end{array}\right)
$$

where 0 is the $1 \times m$ zero matrix, and let $B$ be equal to

$$
B=\left(\begin{array}{ccc}
0 & -g_{3} & g_{2} \\
g_{3} & 0 & -g \\
-g_{2} & g & 0
\end{array}\right) .
$$

If $\mathfrak{A}$ is constructed as in Theorem 3.2, then its generators are the Pfaffians of the skew-symmetric matrix

$$
D=\left(\begin{array}{cc}
B & \tilde{B}_{2} \\
-\tilde{B}_{2}^{t} & \tilde{D}_{2}
\end{array}\right)
$$

Proof. To obtain the result, we make explicit the resolution obtained in Section 3.

First, we show that the maps $\varphi_{1}$ and $\varphi_{2}$ defined in Lemma 3.8 are the null maps, according to our choices for the maps $\beta_{i}$.

By their definition, we have

$$
\Gamma^{\prime} \circ \varphi_{1}=\beta_{1} \circ \bar{\beta}_{2}\left(-t_{I}\right)
$$

and

$$
\wedge^{2} \Gamma^{\prime} \circ \varphi_{2}=\varphi_{1} \circ \wedge^{2} \Gamma\left(-t_{I}+t_{J}\right)-\beta_{2} \circ \bar{\beta}_{1}\left(-t_{I}\right) .
$$

By Remark 3.7 we have $\bar{\beta}_{1}=\beta_{1}^{*} \circ \theta_{2}$ and $\bar{\beta}_{2}=\beta_{2}^{*} \circ \theta_{1}$, where $\theta_{1}$ is represented by the matrix (1) while $\theta_{2}$ is represented by the matrix

$$
\left(\begin{array}{cc}
0 & 1 \\
-1 & 0
\end{array}\right)
$$

Hence, it is a straightforward computation that the maps $\bar{\beta}_{1}$ and $\bar{\beta}_{2}$ are represented by the matrices

$$
\bar{B}_{1}=\left(\begin{array}{c}
1 \\
0 \\
\vdots \\
0
\end{array}\right) \quad \text { and } \quad \bar{B}_{2}=\left(\begin{array}{cc}
0 & 0 \\
-b_{31} & b_{21} \\
\vdots & \\
-b_{3 m} & b_{2 m}
\end{array}\right)
$$

Then, the maps $\beta_{1} \circ \bar{\beta}_{2}\left(-t_{I}\right)$ and $\beta_{2} \circ \bar{\beta}_{1}\left(-t_{I}\right)$ are the null maps, and so we can choose $\varphi_{1}$ and $\varphi_{2}$ as the null maps.

The free resolution of $\mathfrak{A}$ obtained by a mapping cone is

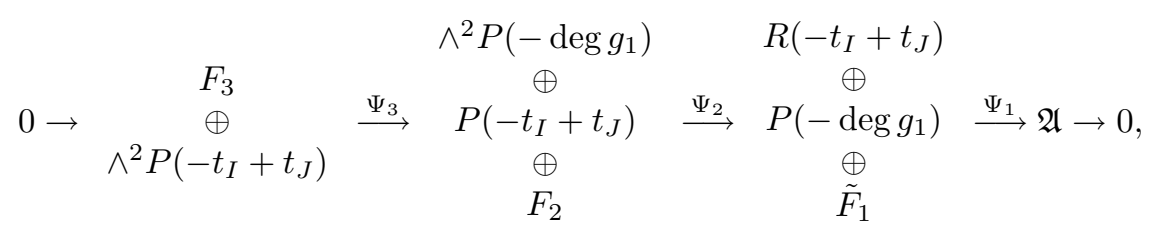


where

$$
\begin{gathered}
\Psi_{1}=\left(\left(f+g g_{1}\right), g_{1} \Gamma^{\prime}, \delta_{1 \mid \tilde{F}_{1}}\right), \\
\Psi_{2}=\left(\begin{array}{ccc}
0 & -\Gamma\left(-t_{I}+t_{J}\right) & 0 \\
\wedge^{2} \Gamma^{\prime} & g \cdot i d & \beta_{2} \\
0 & p \circ \bar{\beta}_{2} & \tilde{\delta}_{2}
\end{array}\right)
\end{gathered}
$$

and

$$
\Psi_{3}=\left(\begin{array}{cc}
-\beta_{3} & -g \cdot i d \\
0 & \wedge^{2} \Gamma\left(-t_{I}+t_{J}\right) \\
\delta_{3} & -\bar{\beta}_{1}
\end{array}\right) .
$$

Of course, the map $\Psi_{1}$ is represented by the matrix

$$
E=\left(f+g g_{1}, g_{1} g_{2}, g_{1} g_{3}, f_{1}, \ldots, f_{m}\right),
$$

whose entries are exactly the generators of $\mathfrak{A}$.

The map $\Psi_{2}$ is represented by the matrix

$$
D^{\prime}=\left(\begin{array}{ccccccc}
0 & -g_{2} & -g_{3} & 0 & 0 & \ldots & 0 \\
g_{3} & g & 0 & 0 & b_{21} & \ldots & b_{2 m} \\
-g_{2} & 0 & g & 0 & b_{31} & \ldots & b_{3 m} \\
0 & -b_{31} & b_{21} & d_{10} & d_{11} & \ldots & d_{1 m} \\
\vdots & & & & & & \\
0 & -b_{3 m} & b_{2 m} & d_{m 0} & d_{m 1} & \ldots & d_{m m}
\end{array}\right),
$$

while the map $\Psi_{3}$ is represented by the matrix

$$
E^{\prime}=\left(\begin{array}{cc}
-f & -g \\
0 & g_{3} \\
0 & -g_{2} \\
g_{1} & -1 \\
f_{1} & 0 \\
\vdots & \\
f_{m} & 0
\end{array}\right)
$$

To cancel the addendum $\wedge^{2} P\left(-t_{I}+t_{J}\right)$ from the resolution we have to modify the matrix $E^{\prime}$ in such a way that one of its rows is $(0,-1)$. To this end, it is enough to add $g_{1}$ times the second column to the first one. Then we obtain the matrix

$$
E^{\prime \prime}=\left(\begin{array}{cc}
-\left(f+g g_{1}\right) & -g \\
g_{1} g_{3} & g_{3} \\
-g_{1} g_{2} & -g_{2} \\
0 & -1 \\
f_{1} & 0 \\
\vdots & \\
f_{m} & 0
\end{array}\right)
$$

If we cancel the addendum in the resolution, we erase the 4-th row and the 2-nd column of $E^{\prime \prime}$ and the 4-th column of $D^{\prime}$. Then, we get the claim by exchanging the rows of $E^{\prime \prime}$ and by changing the signs of its first two entries, so that we have $E^{t}$ as the last matrix in the resolution. Of course, we have to modify the columns of the matrix $D^{\prime}$ according to operations on the rows of $E^{\prime \prime}$. 
Remark 4.6. In the proof of Theorem 4.5, we construct the Buchsbaum-Eisenbud matrix associated to $\mathfrak{A}$ using the matrix $D_{2}$ associated to $I$, the polynomials $g_{2}, g_{3}$ and some relations induced by the inclusion $I \hookrightarrow J$. The main feature of $D$ is

Property P: one of the columns of $D$ has all but two entries equal to zero.

Of course, if $D$ is any skew-symmetric matrix of order $2 k+3$ with the property $P$, we can construct a matrix $D_{2}$ skew-symmetric of order $2 k+1$, using the block structure of $D$ and Lemma 4.1. (2).

Now, we can state the main result of the section.

Theorem 4.7. Let $\mathfrak{A} \subset R$ be a codimension 3 Gorenstein ideal, and let $D$ be a skew-symmetric matrix of order $2 k+3, k>0$, whose Pfaffians are a minimal set of generators for $\mathfrak{A}$. Without loss of generality, assume that $D$ satisfies the property $P$ with respect to the first column. Then, $\mathfrak{A}$ arises from the construction of Theorem 3.2 if, and only if, the ideal I generated by the Pfaffians of $D_{2}$ obtained from $D$ as in Remark 4.6 has codimension 3.

Proof. If $\mathfrak{A}$ arises from the construction of Theorem 3.2 then there exist $I_{1}, J_{1}$ and $g$ which satisfy the hypotheses of Theorem 3.2 By Theorem 4.5, the BuchsbaumEisenbud matrix $D$ of $\mathfrak{A}$ can be constructed from that of $I_{1}$. If we apply the construction of Remark 4.6 to $D$, we get the Buchsbaum-Eisenbud matrix of $I_{1}$, and so we get the "only if" part of the statement with $I=I_{1}$.

To prove the "if" part, we assume that the ideal $I$ generated by the Pfaffians of $D_{2}$ has codimension 3, where $D_{2}$ is obtained by $D$ as in Remark 4.6 From the proof of Theorem 4.5, we know that the Pfaffians of $D$ and $D_{2}$ are related as follows: the last $2 k$ Pfaffians $f_{1}, \ldots, f_{2 k}$ of $D_{2}$ are respectively equal to the last ones of $D$; the first Pfaffian $g_{1}$ of $D_{2}$ is a factor of the second and third Pfaffian $g_{1} g_{2}$ and $g_{1} g_{3}$ of $D$, where $g_{2}$ and $g_{3}$ are the non-zero entries of the first column of $D$; and the first Pfaffian of $D$ has the shape $f+g g_{1}$ for some $f$.

By hypothesis, the ideal $I=\left(g_{1}, f_{1}, \ldots, f_{2 k}\right)$ has codimension 3 , and being Pfaffian, it is Gorenstein. Moreover, $I \subset\left(g_{1}, g_{2}, g_{3}\right)=J$, and hence $J$ has codimension 3 too, i.e., $J$ is a complete intersection ideal. For the same reason, since $\mathfrak{A} \subset\left(f+g g_{1}, g_{2}, g_{3}\right)$, the ideal $\left(f+g g_{1}, g_{2}, g_{3}\right)$ is a complete intersection ideal, where $g=(D)_{23}$. Then, we can apply Theorem 3.2 to $I, J$, and $\left(f+g g_{1}, g_{2}, g_{3}\right)$, and we get exactly the ideal $\mathfrak{A}$ we started with.

Remark 4.8. By [14, Proposition 1.3.7, the general hypersurface sections of an arithmetically Gorenstein scheme are arithmetically Gorenstein, too, and so we can obtain arithmetically Gorenstein schemes of higher codimension by cutting with general hypersurfaces the schemes arising from the construction of Theorem 3.2. These ideals correspond to the same construction for higher codimension, with more than one common generator (compare with Remark 3.11).

\section{COMPARISON With OTHER CONSTRUCTIONS}

In this section we want to compare the Gorenstein codimension 3 ideals arising from Theorem 3.2 with Gorenstein codimension 3 ideals coming from other constructions, well known in the literature.

First, we recall a construction introduced in [19], which has been successfully used by many authors (see, among others, the paper [18]). This construction was studied also in [20]. 
Theorem 5.1 ([14, Theorem 4.2.1). Let $I_{1}, I_{2}$ be Cohen-Macaulay ideals of codimension $c$ with no common minimal associated primes, and assume that $I_{1} \cap I_{2}$ is a Gorenstein ideal. Then, $I_{1}+I_{2}$ is a Gorenstein ideal of codimension $c+1$.

To compare the two constructions, we restrict ourselves to the case where $I_{1}$ and $I_{2}$ have codimension 2 .

Now, we exhibit an example to show that there are Gorenstein ideals arising from the construction of Theorem 3.2 which do not arise from Theorem 5.1

Example 5.2 ([20], Example 4.1). Consider the ideal $\mathfrak{A} \subset R=k[x, y, z, w]$ generated by the Pfaffians of the matrix

$$
D^{\prime}=\left(\begin{array}{ccccc}
0 & x^{4} & 0 & w^{2} & 0 \\
-x^{4} & 0 & w^{3} & x & y \\
0 & -w^{3} & 0 & y & z \\
-w^{2} & -x & -y & 0 & 0 \\
0 & -y & -z & 0 & 0
\end{array}\right)
$$

As explained in [20], Example 4.1, $\mathfrak{A}$ does not arise from the construction of Theorem [5.1] but it does come from the construction of Theorem 3.2. In fact, if we reorder rows and columns of $D^{\prime}$, we obtain the matrix

$$
D=\left(\begin{array}{ccccc}
0 & -y & -z & 0 & 0 \\
y & 0 & w^{3} & x & -x^{4} \\
z & -w^{3} & 0 & y & 0 \\
0 & -x & -y & 0 & -w^{2} \\
0 & x^{4} & 0 & w^{2} & 0
\end{array}\right)
$$

As in Remark 4.6, we construct the matrix $\Delta$ from $D$, and we obtain

$$
\Delta=\left(\begin{array}{ccc}
0 & y^{2}-x z & x^{4} z \\
-y^{2}+x z & 0 & -w^{2} \\
-x^{4} z & w^{2} & 0
\end{array}\right) .
$$

The ideal generated by the Pfaffians of $\Delta$ is $I=\left(w^{2}, z x^{4}, y^{2}-x z\right)$, and it is a complete intersection ideal of codimension 3 . Then, $\mathfrak{A}$ satisfies the hypotheses of Theorem 4.7, and so $\mathfrak{A}$ arises from the construction of Theorem 3.2 .

On the contrary, now we give an example to show that there are Gorenstein ideals arising from the construction of Theorem 5.1 which cannot be obtained from the construction of Theorem 3.2 .

In [20], Theorem 4.3, the authors prove that every arithmetically Gorenstein scheme $X$ on a smooth quadric $Q$ comes from Theorem 5.1. In particular, they prove that $I_{X}$, the saturated ideal of $X$, is minimally generated by 5 generators of degrees $2, a, a, b, b$ with $2 \leq a \leq b$, and $I_{X}=I_{C}+I_{D}$, where, if $C$ is a divisor on $Q$ of type $(a-1, a)$, then $D$ is a divisor of type $(b, b-1)$. Of course, the types could be $(a, a-1)$ and $(b-1, b)$, as well.

Example 5.3. Let $Q \subset \mathbb{P}^{3}$ be the smooth quadric surface defined by $x w-y z$, and let $C, D$ be divisors on $Q$ defined by $I_{1}=\left(x w-y z, z^{2}-y w, y^{2}-x z\right)$ and $I_{2}=\left(x w-y z, w z^{2}-y x^{2}, x^{3}-z^{3}\right) . C$ is a twisted cubic, and so it is irreducible, and it is not a component of $D$. Then, $C$ and $D$ have no common component. Furthermore $C \cup D$ is the complete intersection scheme defined by

$$
J=\left(x w-y z, x^{3} z-z^{4}-y^{2} x^{2}+y z^{2} w\right) .
$$


Then, $C \cap D$ is an arithmetically Gorenstein scheme associated to the skewsymmetric matrix

$$
D=\left(\begin{array}{ccccc}
0 & z^{2} & x^{2} & y & z \\
-z^{2} & 0 & 0 & x & y \\
-x^{2} & 0 & 0 & z & w \\
-y & -x & -z & 0 & 0 \\
-z & -y & -w & 0 & 0
\end{array}\right)
$$

Neither $D$ nor any matrix obtained from $D$ by operations on its rows and columns satisfies the hypotheses of Theorem 4.7, and so $I_{X}$ cannot be obtained from the construction of Theorem 3.2 ,

Remark 5.4. It may happen that a Gorenstein codimension 3 ideal arises from both constructions. For example, a reduced set $X \subset \mathbb{P}^{3}$ of five general points can be obtained both as an intersection of two aCM curves whose union is a complete intersection (for example, choose $C$ as a divisor of type $(1,2)$ and $D$ as a divisor of type $(2,1)$ on a smooth quadric $Q$ ), and as the union of a complete intersection with a residual scheme (for example, consider the residual of a complete intersection of type $(1,1,1)$ with respect to a complete intersection of type $(1,2,2)$ joined with a complete intersection of type $(1,1,2))$. For more details on the second construction, see [1] or Example 3.18.

In more generality, for zero dimensional arithmetically Gorenstein schemes on a smooth quadric, we have

Proposition 5.5. Let $Q \subset \mathbb{P}^{3}$ be a smooth quadric surface, and let $X \subset Q$ be an arithmetically Gorenstein 0-dimensional scheme. If $I_{X}$ comes from the construction of Theorem 3.2. then $X=C \cap D$, where $C, D$ are divisors on $Q$ and at least one of them is the union of a line and a divisor of type $(\alpha, \alpha)$.

Proof. By [20], Proposition 4.4, $I_{X}$ is generated by the Pfaffians of a skew-symmetric matrix

$$
D=\left(\begin{array}{ccccc}
0 & h_{1} & h_{2} & h_{3} & h_{4} \\
-h_{1} & 0 & 0 & g_{1} & g_{2} \\
-h_{2} & 0 & 0 & g_{3} & g_{4} \\
-h_{3} & -g_{1} & -g_{3} & 0 & 0 \\
-h_{4} & -g_{2} & -g_{4} & 0 & 0
\end{array}\right),
$$

where $q=g_{1} g_{4}-g_{2} g_{3}$ defines the smooth quadric surface $Q$.

Up to some elementary operations on rows and columns of $D$, we can suppose that at least one of the $h_{i}$ 's is zero, because $I_{X}$ can be obtained from Theorem 4.7 Assume $h_{4}=0$. Then, either $C$ or $D$ is defined by

$$
I=\left(q, h_{3} g_{2}, h_{3} g_{4}\right)=\left(q, h_{3}\right) \cap\left(g_{2}, g_{4}\right),
$$

and so the claim follows.

Remark 5.6. 1) The converse holds if the matrix $D$ satisfies the hypotheses of Theorem 4.7, i.e., if $h_{3}, h_{1} g_{4}-h_{2} g_{2}, g_{2}^{2}-g_{1} g_{4}$ is a regular sequence.

2) A similar statement holds for 0-dimensional arithmetically Gorenstein schemes on a smooth surface of minimal degree, whose ideal is minimally generated by five forms. The proof is the same and is based on [20], Proposition 4.4. 
The second construction with which we want to compare the construction of Theorem 3.2 was introduced in [12, and used by many authors to study Gorenstein liaison classes of projective schemes (see, among others, [5], [6], [7], [13]).

Theorem 5.7 ([14], Theorem 4.2.8). Let $S$ be an aCM subscheme of $\mathbb{P}^{n}$ satisfying condition $G_{1}$, and let $X$ be a twisted canonical divisor on $S$ (i.e., a subscheme of $S$ defined by the vanishing of a regular section of $\omega_{S}(l)$ for some $\left.l \in \mathbb{Z}\right)$. Let $F \in I_{X}$ be a homogeneous polynomial of degree $d$ such that $F$ does not vanish on any component of $S$. Let $H_{F}$ be the divisor cut out on $S$ by $F$. Then the (effective) divisor $H_{F}-X$ on $S$, viewed as a subscheme of $\mathbb{P}^{n}$, is arithmetically Gorenstein. In fact, any effective divisor in the linear system $\left|H_{F}-X\right|$ is arithmetically Gorenstein.

As before, to show that the constructions of Theorems 3.2 and 5.7 are independent, we exhibit examples of Gorenstein codimension 3 ideals arising from one of the two constructions but not from the other one.

Example 5.8. In $S=k[x, y, z]$, we consider the complete intersection ideals $J=$ $(x, y, z)$ and $I=\left(x^{2}-z^{2}, x z, y\right)$. Of course, $I \subset J$ and $I: J=\left(x^{2}-z^{2}, x z, y, x^{2}\right)=$ $\left(x^{2}, x z, z^{2}, y\right)$. The ideal $\left(x, z, x^{2}-y^{2}\right)$ is a complete intersection ideal, and so $(I: J) \cap\left(x, z, x^{2}-y^{2}\right)=\left(x^{2}-z^{2}, x z, y z, x y, y^{2}-z^{2}\right)=\mathfrak{A}$ is a codimension 3 Gorenstein ideal obtained as in Theorem [3.2 Let $R=S[w]$, and by abuse of notation let $\mathfrak{A}$ be the extended ideal $\mathfrak{A} R$. The projective scheme $X$ defined by $\mathfrak{A}$ in $\mathbb{P}^{3}$ is a degree 50 -dimensional scheme supported on $A(0: 0: 0: 1)$. $X$ is not contained in any smooth quadric (and so not in any rational normal curve nor any degeneration of such a curve with the property $G_{1}$ ). On the other hand, $X$ is contained in the union of three lines $L_{1}=V(y, x+z), L_{2}=V(y, x-z), L_{3}=V(x, z)$ through the point $A$. As observed in [14, Example 4.2.7, $L_{1} \cup L_{2} \cup L_{3}$ doesn't satisfy $G_{1}$ but only $G_{0}$, and so Theorem 5.7 doesn't apply.

Remark 5.9. As shown in Example 5.8. Theorem 3.2 can be used to construct Artinian Gorenstein codimension 3 ideals, as well.

An example which shows that there are arithmetically Gorenstein schemes arising from Theorem 5.7 and not from Theorem 3.2 is the previous Example 5.3 . In the setting of Theorem 5.7, $C$ is a twisted cubic curve, and so it is aCM, smooth, with the property $G_{1}$, and $C \cap D$ is a 0-dimensional scheme of degree 8. $C \cap D$ is in the linear system $\left|H_{F}-X\right|$, where $F=x^{3}-z^{3}$ and $X=(0: 0: 0: 1)$ with the reduced scheme structure. As previously observed, $C \cap D$ cannot be obtained from the construction of Theorem 3.2 .

The third construction with which we compare Theorem 3.2 is the following.

Theorem 5.10 ([14], Theorem 4.2.3). Let $s$ be a regular section of a BuchsbaumRim sheaf $\mathcal{B}_{\phi}$ of rank $r$ on $\mathbb{P}^{n}$, and let $I$ be the ideal corresponding to s. Let $Y$ be the subscheme of $\mathbb{P}^{n}$ defined by $I$. Let $J$ be the top dimensional component of $I$, defining a scheme $X$.

(a) If $r=n$, then $X=Y$ is a zero scheme and $J$ is the saturation of $I$.

(b) If $r$ is even, $r \leq n$, then $I=J$ is saturated and $Y=X$ is arithmetically Cohen-Macaulay. Its Cohen-Macaulay type is $\leq 1+\left(\begin{array}{c}r / 2+t-1 \\ t-1\end{array}\right)$.

(c) If $r$ is odd, $r \leq n$, then $I$ is not saturated. If $r<n$, then $Y$ is not arithmetically Cohen-Macaulay. However, the top dimensional part, $X$, is arithmetically Gorenstein. 
This construction, presented in [15], was further investigated in [16].

In [15], Example 5.3, the authors construct a smooth 3-fold in $\mathbb{P}^{6}$ using Theorem 5.10. Of course, using Theorem [3.2] it is not possible to obtain smooth schemes, because if they are irreducible then they are not reduced. However, we give an example of a zero-dimensional scheme in $\mathbb{P}^{3}$ constructed using Theorem 5.10 which cannot be constructed using Theorem 3.2

Example 5.11. Let $\mathcal{B}_{\phi}$ the Buchsbaum-Rim sheaf associated to the map $\phi$ : $\mathcal{O}_{\mathbb{P}^{3}}^{4}(-1) \rightarrow \mathcal{O}_{\mathbb{P}^{3}}$ defined by $(x, y, z, t)$. Of course, the cokernel of $\phi$ has codimension 4. The module $B_{\phi}=H_{*}^{0}\left(\mathbb{P}^{3}, \mathcal{B}_{\phi}\right)$ is generated by the columns of the matrix associated to $\wedge^{2} \phi$. We consider the ideal $I$ associated to the regular section $s$ given by $\left(t^{2}, z t,-z^{2}, y z, x y, x^{2}\right)$. The top dimensional part (or its saturation, in this case) is defined by the ideal $J$ generated by $x^{3}-x y z-z t^{2}, x^{2} y+y z^{2}+t^{3}, x y^{2}+x y z+$ $z^{2} t, z^{3}-y z t-x t^{2}, x^{2} z^{2}+y z^{2} d-x y t^{2}$, which is arithmetically Gorenstein, with h-vector $(1,3,6,6,3,1)$. The Buchsbaum-Eisenbud matrix of $J$ is

$$
\left(\begin{array}{ccccc}
0 & z^{2} & -t^{2} & -y z & -y \\
-z^{2} & 0 & -z t & -x y & x \\
t^{2} & z t & 0 & x^{2} & -z \\
y z & x y & -x^{2} & 0 & t \\
y & -x & z & -t & 0
\end{array}\right)
$$

which doesn't satisfy property P. Hence, $J$ cannot be obtained using Theorem 3.2

Now, we give an example to show that there are arithmetically Gorenstein ideals constructed using Theorem 3.2 which cannot be constructed using Theorem 5.10 ,

Example 5.12. In [15, Remark 5.1, the authors prove that the ideal

$$
J=\left(x^{2}, y^{2}, z^{2}, x y-y z, x z-y z\right)
$$

cannot be obtained from Theorem 5.10 .

The ideals $I=\left(z^{2}, x^{2}+x y-y z, x-y\right)$ and $J^{\prime}=(z, x+y, x-y)$ are complete intersection ideals of codimension 3 , with a common generator, and $I \subset J^{\prime}$. By applying Theorem 3.2 to them, with $g=x-z$, we get $\mathfrak{A}=J$, and so we obtain the independence of the constructions of Theorems 3.2 and 5.10 .

As our last comparison, using notation as in Theorem 4.7, we show that $\mathfrak{A}$ is not a basic double G-linkage either of $I$ or of $I_{\text {res }}$. For the definition of basic double G-linkage see, for example, [14, Definition 5.4.6.

It is evident that $\mathfrak{A}$ is not a basic double G-linkage of $I$, because $\mathfrak{A} \nsubseteq I$, while in a basic double G-linkage the resulting ideal is contained in the starting one.

To prove that $\mathfrak{A}$ is not a basic double G-linkage of $I_{\text {res }}$, we consider the following example.

Example 5.13. Let $\mathbb{P}^{3}=\operatorname{Proj}(k[x, y, z, w])$ and let $S$ be the aCM curve defined by $I_{S}=(x, y) \cap(x, z) \cap(z, w) . S$ satisfies the property $G_{1}$. Let

$$
I=(x, y z, w(y-w)(2 y-w)(y+w))
$$

be a complete intersection ideal, contained in $J=(x, z, w)$. The residual ideal $I: J$ is

$$
I_{\mathrm{res}}=(x, y z, w(y-w)(y+w)(2 y-w), y(y-w)(y+w)(2 y-w)),
$$

and the corresponding scheme $C$ has degree 7. By applying Theorem [3.2] we obtain an arithmetically Gorenstein scheme of degree 11, contained in $S$. This scheme is 
not a basic double G-linkage of $C$ on $S$ for degree reasons, because the degree of every basic double G-linkage of $C$ on $S$ is $\equiv 7(\bmod 3)$.

Remark 5.14. In Example 5.8, we constructed an Artinian Gorenstein codimension 3 ideal $\mathfrak{A}=\left(x^{2}-z^{2}, x z, y z, x y, y^{2}-z^{2}\right) \supseteq(x, y, z)^{3}$, in $k[x, y, z]$. If we extend it in $R=k[x, y, z, t, u]$, we obtain schemes which are not $G_{0}$, and so our construction can be applied in the non- $G_{0}$ case, too.

\section{ACKNOWLEDGEMENTS}

During Pragmatic 2000, the first two authors were asked by A.V. Geramita and J.C. Migliore to find a geometrical construction of reduced arithmetically Gorenstein zero-dimensional schemes in $\mathbb{P}^{3}$, in such a way as to obtain "visible" configurations of points. The resulting paper [1] is the starting point of our construction. Therefore we all want to warmly thank A.V. Geramita and J.C. Migliore for suggesting such an interesting topic and for encouraging the development of the present work in various enlightening discussions.

The calculations underlying most examples and leading to the discovery of several of the propositions were carried out using the computer algebra system CoCoA (cf. 4]).

\section{REFERENCES}

[1] C. Bocci, G. Dalzotto, Gorenstein points in $\mathbb{P}^{3}$, Rend. Sem. Mat. Univ. Politec. Torino 59 (2001) n. 1, 155-164.

[2] W. Bruns, J. Herzog, "Cohen-Macaulay Rings", Cambridge Studies in Adv. Math. 39, Cambridge University Press, 1993. MR 95h:13020

[3] D. Buchsbaum, D. Eisenbud, Algebra structures for finite free resolutions and some structure theorems for ideals of codimension three, Amer. J. Math. 99 (1977), 447-485. MR 56:11983

[4] A. Capani, G. Niesi, L. Robbiano, CoCoA, a System for Doing Computations in Commutative Algebra, Version 4.2 is available at http://cocoa.dima.unige.it.

[5] M. Casanellas, R.M. Miró-Roig, Gorenstein liaison of curves in $\mathbb{P}^{4}$, J. Algebra 230 (2000), 656-664. MR 2001g:14082

[6] M. Casanellas, R.M. Miró-Roig, Gorenstein liaison of divisors on standard determinantal schemes and on rational normal scrolls, Journal of Pure and Applied Algebra 164 (2001), 325-343. MR 2002g:14071

[7] M. Casanellas, R.M. Miró-Roig, Gorenstein liaison and special linear configurations, Illinois Math. J. 46 (2002), 129-143 MR 2003i:14062

[8] E. Davis, A.V. Geramita, F. Orecchia, Gorenstein Algebras and the Cayley-Bacharach Theorem, Proceedings AMS 93, n. 4 (1985), 593-597. MR 86k:14034

[9] S. Diesel, Irreducibility and Dimension Theorems for Families of Height 3 Gorenstein Algebras, Pacific J. Math. 172 (1996), n. 2, 365-397. MR 99f:13016

[10] A.V. Geramita, J.C. Migliore, Reduced Gorenstein codimension three subschemes of projective schemes, Proc. Amer. Math. Soc. 125 (1997), 943-950. MR 97h:14068

[11] R. Hartshorne, Some examples of Gorenstein liaison in codimension three, Collectanea Math. 53 (2002), 21-48. MR 2003d:14059

[12] J.O. Kleppe, J.C. Migliore, R.M. Miró-Roig, C. Peterson, Gorenstein Liaison, Complete Intersection Liaison Invariants and Unobstructedness, Mem. Amer. Math. Soc. 154, n. 732, (2001). MR 2002e:14083

[13] J. Lesperance, Gorenstein liaison of some curves in $\mathbb{P}^{4}$, Collectanea Math. 52 (2001), 219230. MR 2003g:14065

[14] J.C. Migliore, "Introduction to Liaison Theory and Deficiency Modules", Progress in Mathematics 165, Birkhäuser, 1998. MR 2000g:14058

[15] J.C. Migliore, C. Peterson, A construction of codimension three arithmetically Gorenstein subschemes of projective space, Trans. Amer. Math. Soc. 349 (1997), 3803-3821. MR 98d:14060 
[16] J.C. Migliore, U. Nagel, C. Peterson, Buchsbaum-Rim sheaves and their multiple sections, J. Algebra 219 (1999), 378-420. MR 2000f:14076

[17] J.C. Migliore, U. Nagel, Monomial ideals and the Gorenstein liaison class of a complete intersection, Compositio Math. 133 (2002), n. 1, 25-36. MR 2003g:13010

[18] J.C. Migliore, U. Nagel, Reduced arithmetically Gorenstein schemes and simplicial polytopes with maximal Betti numbers, Adv. Math. (to appear)

[19] C. Peskine, L. Szpiro, Liaison des variétés algébriques I, Inv. Math. 26 (1974), 271-302. MR $51: 526$

[20] A. Ragusa, G. Zappalà, Properties of 3-codimensional Gorenstein schemes, Comm. Alg. 29 (2001), n. 1, 303-318. MR 2002f:14061

[21] R. Stanley, Hilbert functions of graded algebras, Adv. in Math. 28 (1978), 57-82. MR 58:5637

[22] J. Watanabe, A note on Gorenstein rings of embedding codimension three, Nagoya Math. J. 50 (1973), 227-232. MR 47:8526

Dipartimento di Matematica, Università di Torino, I-10123 Torino, Italy

E-mail address: bocci@dm.unito.it

Current address: Dipartimento di Matematica, Università di Milano, I-20133 Milano, Italy

E-mail address: cristiano.bocci@unimi.it

Dipartimento di Matematica, Universitì di Genova, I-16146 Genova, Italy

E-mail address: dalzotto@module.dima.unige.it

Current address: Dipartimento di Matematica, Università di Pisa, I-56127 Pisa, Italy

E-mail address: dalzotto@mail.dm.unipi.it

Dipartimento di Matematica, Politecnico di Torino, I-10129 Torino, Italy

E-mail address: roberto.notari@polito.it

Dipartimento di Matematica, Politecnico di Torino, I-10129 Torino, Italy

E-mail address: maria.spreafico@polito.it 